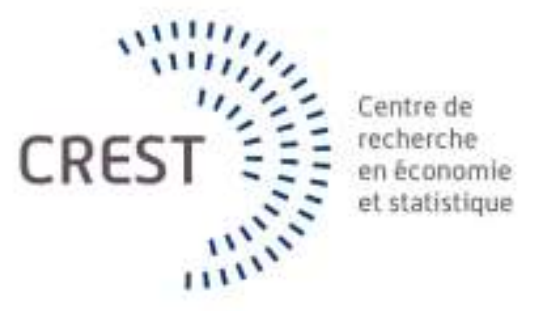

Série des Documents de Travail

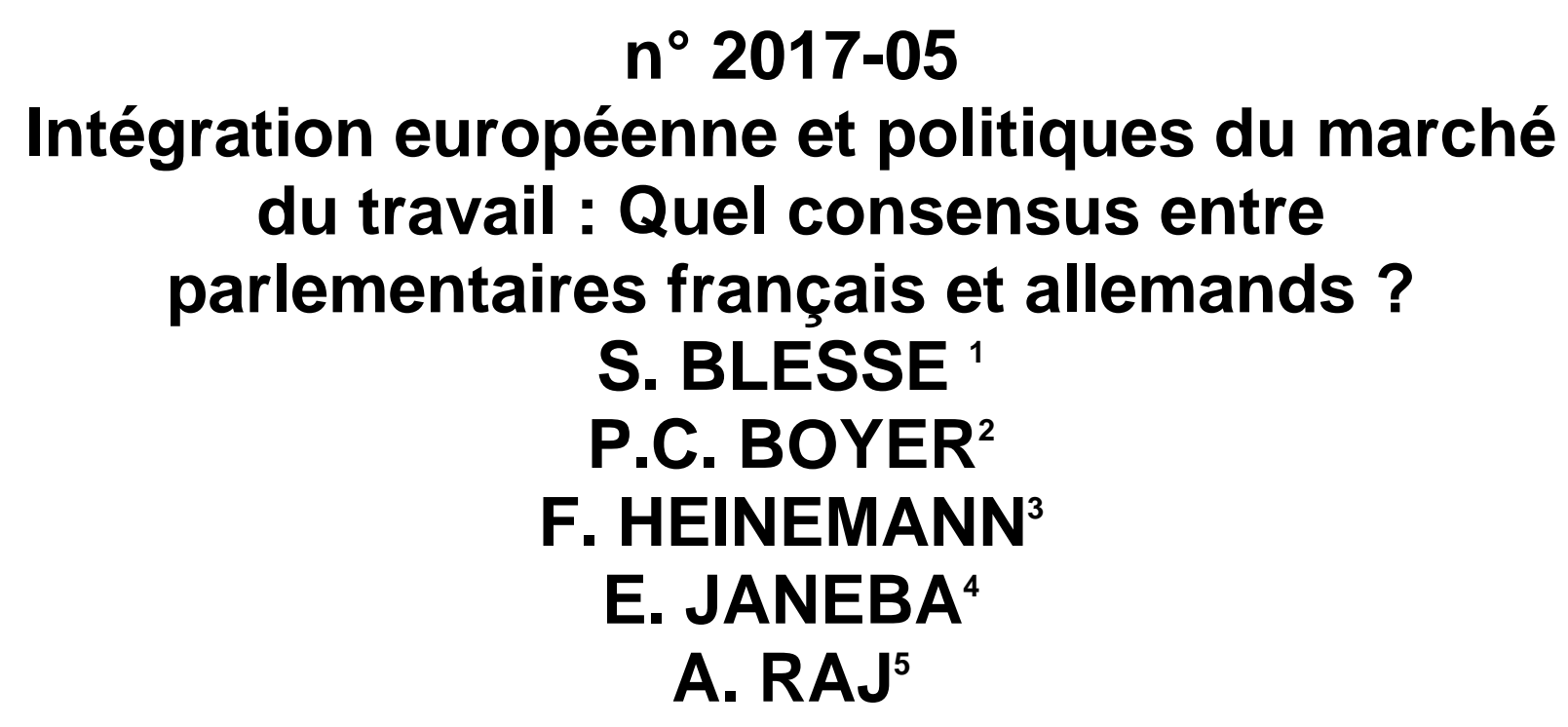

Les documents de travail ne reflètent pas la position du CREST et n'engagent que leurs auteurs. Working papers do not reflect the position of CREST but only the views of the authors.

\footnotetext{
${ }^{1}$ ZEW, Department Corporate Taxation and Public Finance, Allemagne

${ }^{2}$ CREST, École Polytechnique, Université Paris-Saclay, France

${ }^{3}$ Université de Heidelberg ; ZEW, Department Corporate Taxation and Public Finance, Allemagne

${ }^{4}$ Université de Mannheim, Allemagne

${ }^{5}$ CREST, École Polytechnique, Université Paris-Saclay, France
} 


\title{
Intégration européenne et politiques du marché du travail : Quel consensus entre parlementaires français et allemands ?*
}

\author{
Sebastian Blesse ${ }^{\mathrm{a}}$, Pierre C. Boyer ${ }^{\mathrm{b}}$, Friedrich Heinemann ${ }^{\mathrm{c}}$, Eckhard Janeba ${ }^{\mathrm{d}}$ \\ et Anasuya Raj ${ }^{b}$ \\ a ZEW, Department Corporate Taxation and Public Finance, Allemagne \\ ${ }^{\mathrm{b}}$ CREST, École Polytechnique, Université Paris-Saclay, France \\ ${ }^{\mathrm{c}}$ Université de Heidelberg; ZEW, Department Corporate Taxation and Public Finance, Allemagne \\ ${ }^{\mathrm{d}}$ Université de Mannheim, Allemagne
}

24 mai 2017

\begin{abstract}
Résumé
Cet article présente les résultats d'une enquête menée auprès des parlementaires français et allemands sur l'opportunité d'une intégration européenne dans les domaines liés au marché du travail. En particulier, l'avis de parlementaires est recueilli sur l'intérêt d'une plus grande intervention de l'Union Européenne dans la règlementation du marché du travail, la politique salariale et la politique d'immigration dans les États membres. Ils ont également exprimé leur opinion sur la création d'une assurance chômage commune européenne, ou sur la nécessité de rendre le marché du travail plus flexible. Après une présentation descriptive des réponses, nous montrons que pour la majorité des questions nos résultats font état d'un clivage partisan gauche/droite plus marqué qu'une différence France/Allemagne.
\end{abstract}

Mots-clés : Intégration européenne; enquête ; parlementaires nationaux français et allemands; politiques du marché du travail.

Codes de classification JEL : F15; J40 ; J60 ; J80 ; C83.

\footnotetext{
${ }^{*}$ Nous tenons à remercier Lionel Wilner pour ses commentaires. Nous remercions également la Fondation allemande pour la recherche dans le cadre du programme interdisciplinaire sur l'Économie politique des réformes à l'Université de Mannheim (programme SFB 884) et les Investissements d'Avenir (ANR-11-IDEX0003/Labex Ecodec/ANR-11-LABX-0047) pour leurs soutiens financiers. Courriel (auteur correspondant) : pierre.boyer@polytechnique.edu
} 


\title{
European integration and labor market policies: Is there a consensus between French and German members of national parliaments?
}

\begin{abstract}
This paper presents the results of a survey on the future of European integration conducted in the national parliaments of both France and Germany. In particular, we collected the opinions of members of national parliaments on more involvement of the European Union in labor market and immigration policies of Member States. We also asked them about their views on the creation of a common unemployment insurance and whether more flexibility is needed in national labor markets. After reporting descriptive and summary statistics, we show that for the majority of questions the answers reflect more of an ideological (left/right) division rather than a French/German one.
\end{abstract}

Keywords: European integration; survey; French and German members of national parliament; labor market policies.

JEL Classification: F15; J40; J60; J80; C83. 


\section{Introduction}

La série de crises qui touchent la construction de l'Union Européenne (UE) depuis 2005 avec le rejet du traité constitutionnel en France et aux Pays-Bas, et dont la décision du Royaume-Uni de quitter l'UE en juin 2016 est le dernier épisode, ont mis au premier plan la nécessité d'un débat sur les compétences à allouer à l'échelon européen. Les suggestions de réformes sont nombreuses, allant de stratégies visant à une union fédéraliste conférant plus de compétences politiques au niveau européen à des stratégies de désintégration, donnant plus d'autonomie au niveau national. ${ }^{1}$

La recherche de légitimité pour les décisions de l'UE ou de la zone euro est souvent associée à une nécessité de consensus large et d'une implication plus forte des parlements nationaux. ${ }^{2}$ Ainsi nous avons recueilli l'opinion de parlementaires français de l'Assemblée nationale et du Sénat, et de parlementaires allemands du Bundestag sur l'élargissement des compétences de l'UE. Contrairement aux analyses régulières faites sur les préférences en matière d'intégration des citoyens européens (cf. les différentes vagues de l'Eurobaromètre depuis 1974), un manque de données persiste sur l'opinion des responsables politiques face à une évolution du projet européen. L'analyse économétrique des réponses présentée dans cet article contribue donc à enrichir les grands débats contemporains sur l'intégration européenne.

Un consensus des parlementaires des deux côtés du Rhin ne garantit certainement pas l'acceptation de propositions d'intégration par le reste des membres de l'UE. Cependant, étant donné l'importance historique de ces deux nations dans le processus d'intégration européenne et la difficulté d'obtenir une majorité qualifiée au Conseil de l'Union Européenne sans l'aval d'au moins un des deux pays, il est probable que des réformes soutenues ni par le parlement français ni par le parlement allemand auraient peu de chances d'être adoptées. Nous voyons donc un consensus franco-allemand comme une condition nécessaire pour la faisabilité de réformes liées à l'intégration européenne.

Notre enquête recueille l'avis de parlementaires sur plusieurs questions liées à l'intégration européenne à travers une série de questions spécifiques sur les instruments et options pour y parvenir. Elle a été menée entre avril et juillet 2016. Nous présentons dans cet article les résultats des questions se rattachant à la thématique du marché du travail. ${ }^{3}$

Les politiques liées au marché du travail sont un volet essentiel dans la politique des États membres et elles sont soumises à une attente de résultats forte des citoyens vis-à-vis de leurs représentants (les citoyens placent régulièrement l'emploi parmi leurs préoccupations

\footnotetext{
${ }^{1}$ Pour une analyse coûts/bénéfices de l'intégration fédérale dans la littérature académique voir par exemple Alesina et al. (2005). Cette littérature est inspirée de la littérature de fédéralisme fiscal, cf. Oates (1999).

${ }^{2}$ Voir Rosanvallon (2013) pour une histoire et une théorie de la légitimité démocratique.

${ }^{3}$ Un résumé non-technique présentant les résultats de l'enquête peut être trouvé dans une note de politique du ZEW, cf. Blesse et al. (2016).
} 
essentielles de la vie quotidienne). De plus, dans un sondage Eurobaromètre spécial du Parlement européen en avril 2016, les citoyens européens placent la lutte contre le chômage et la question migratoire parmi les domaines où ils souhaitent que l'UE intervienne davantage. Des réformes dans ce domaine sont régulièrement faites (cf. Turrini et al., 2015) mais elles restent dans le giron des États membres et soulèvent de vives critiques. ${ }^{4}$ La perception des parlementaires sur l'opportunité d'allouer plus de compétences à l'UE dans ce domaine est donc particulièrement intéressante.

Les parlementaires ont donné leur avis à propos des questions qui sont pour les trois premières directement liées à un soutien/opposition à plus d'intégration européenne des domaines clés puis, pour les deux dernières, des propositions qu'ils ont approuvées/rejetées (échelle des réponses de -4 à 4,0 étant libellé comme Indifférent) :

1. Marché du travail : "L'UE pourrait donner des directives contraignantes aux États membres relatives au marché du travail (par exemple sur les modalités de la protection des salariés contre les licenciements ou sur les clauses du contrat de travail à durée déterminée)".

2. Politique salariale : "L'UE pourrait avoir des droits d'intervention plus importants dans les politiques salariales des États membres (par exemple sur le niveau du salaire minimum légal)".

3. Politique d'immigration : "L'UE devrait jouer un rôle renforcé dans la politique d'immigration des États membres (par exemple en fixant les normes standard d'accueil ou en décidant de la répartition des réfugiés)".

4. Assurance chômage européenne : "Pour pallier une éventuelle récession de certains États membres dans la zone euro il faudrait créer une assurance chômage commune européenne".

5. Marché du travail plus flexible : "Une croissance plus forte dans la zone euro requière que les États comptabilisant un nombre important de chômeurs de longue durée rendent plus flexible leur marché du travail (par exemple en assouplissant les conditions de licenciement ou en baissant le niveau du salaire minimum)".

Notre analyse utilise un modèle Probit ordonné. ${ }^{5}$ De façon générale, nos résultats montrent un non-alignement des préférences exprimées sur l'intégration européenne entre les membres

\footnotetext{
${ }^{4}$ Voir par exemple Saint-Paul (1996, 2002) et Boeri et al. (2012) pour une économie politique des réformes du marché du travail. Voir également le manuel de Cahuc et al. (2014) pour une revue des problématiques liées au marché du travail.

${ }^{5}$ Les résultats obtenus sont robustes lorsque l'on change de modèle ou que l'on considère d'autres variables, voir la section 4 .
} 
des partis de droite et de gauche. Ce clivage gauche/droite est souvent plus marqué que la différence France/Allemagne.

Pour les deux premières questions, nous obtenons un effet significatif pour la nationalité et l'appartenance politique. Les deux effets sont tels que la probabilité d'être plus en faveur de la proposition indiquée ceteris paribus augmente avec le fait d'être français d'une part et d'appartenir à un parti de gauche d'autre part. Cependant les effets des deux variables sont du même ordre de grandeur pour la première question alors que la nationalité a un plus fort effet que l'affiliation politique pour la politique salariale.

La troisième question s'intéresse à la question de l'immigration. Nous avons décidé d'inclure cette question car l'immigration joue souvent un rôle clé dans le fonctionnement du marché du travail. ${ }^{6}$ Nous obtenons un effet significatif pour la nationalité et l'appartenance politique. Les deux effets sont tels que ceteris paribus être français diminue la probabilité d'être en faveur de la proposition et appartenir à un parti de gauche augmente la probabilité d'être plus en faveur de la proposition. L'effet de la nationalité est très fort : les allemands sont très favorables à une intégration européenne dans ce domaine. Les parlementaires français membres de partis de droite sont les plus réservés.

L'introduction d'une assurance chômage commune européenne est une piste pour introduire un mécanisme de partage des risques affectant les pays membres en cas de chocs touchant différemment les pays en temps de crises (voir Dolls et al., à paraître). Notre analyse montre que la probabilité d'être plus en faveur de la proposition indiquée ceteris paribus augmente avec le fait d'être français d'une part et d'appartenir à un parti de gauche d'autre part. Les effets ne sont pas du même ordre de grandeur : le clivage politique est beaucoup plus fort que celui lié la nationalité. Ces réponses données montrent qu'un clivage partisan s'oppose à une allocation au niveau européen des mécanismes de partage des risques sur les marchés du travail nationaux.

Enfin, les réformes structurelles visant à plus de flexibilité du marché du travail représentent typiquement une politique d'offre. Cette question révèle un clivage exclusivement partisan : la probabilité d'être plus en faveur de la proposition indiquée ceteris paribus augmente fortement avec le fait d'appartenir à un parti de droite. Une forte polarisation gauche/droite est donc observée alors que la nationalité du parlementaire ne joue pas un rôle significatif dans les réponses à cette question.

La section suivante présente les données. Ensuite, nous analysons les résultats du modèle Probit ordonné. Dans la section 4, nous procédons à des tests de robustesse. La dernière section conclut l'analyse économétrique.

\footnotetext{
${ }^{6}$ Par exemple, l'immigration au sein d'une union monétaire est un facteur important pour la stabilité macroéconomique, cf. Farhi and Werning (2014).
} 


\section{Les données}

Nos données proviennent d'une enquête menée par l'École Polytechnique, le ZEW (Centre pour la Recherche Européenne en Économie, Zentrums für Europäische Wirtschaftsforschung) et l'Université de Mannheim. Cette enquête a été financée par la Fondation allemande pour la recherche (Deutsche Forschungsgemeinschaft) dans le cadre du programme interdisciplinaire sur l'Économie politique des réformes à l'Université de Mannheim (programme SFB 884).

Description de l'enquête. Les questionnaires ont été envoyés par courrier en France et en Allemagne à la fin du mois d'avril 2016. Les trois pages du questionnaire, accompagnées d'une lettre explicative, ont été envoyées au même moment à l'adresse principale de chaque parlementaire, depuis Palaiseau pour les parlementaires français (Assemblée Nationale et Sénat) et depuis Mannheim pour les parlementaires allemands (Bundestag). ${ }^{7}$ Les questionnaires, en français et en allemand, ont le même contenu. Quatre possibilités ont été offertes aux parlementaires pour y répondre (courrier, courriel, fax ou en ligne). Des courriels de relance ont été envoyés quelques semaines plus tard à ceux qui n'avaient pas encore répondu et qui n'avaient pas explicitement indiqué ne pas vouloir participer à l'enquête. Enfin, des relances téléphoniques ont eu lieu en juin et juillet pour les parlementaires restants. Les questionnaires remplis ont été reçus entre la fin du mois d'avril et la mi-juillet 2016.

Afin de pouvoir utiliser les caractéristiques individuelles des parlementaires (parti politique, âge, sexe, etc.), nous avons décidé que l'enquête ne serait pas anonyme. Cependant, pour encourager les parlementaires à exprimer sans biais leurs préférences, la confidentialité des réponses personnelles leur a été garantie.

Le questionnaire se divise en trois parties : la répartition des missions et compétences en Europe, le pacte budgétaire européen, et la politique monétaire et des finances dans la zone euro. Les réponses possibles à l'enquête s'échelonnent selon une échelle de Likert entre -4 (Pas d'accord) et +4 (D'accord). Le 0 a été libellé comme Indifférent. ${ }^{8}$

Sur les 1552 questionnaires envoyés, 232 ont été complétés (le taux de réponse est donc de $14,95 \%)$. Le taux de réponses est de 14,21\% pour les parlementaires français $(15,27 \%$ pour les sénateurs et sénatrices et 13,57\% pour les député-e-s) et de 16,03\% pour les parlementaires allemands. Sur les 232 parlementaires ayant répondu, 101 sont allemands (sur 630) et 131 sont français (sur 922).

Afin de faciliter une comparaison franco-allemande, nous avons classifié les partis nationaux selon l'affiliation de leurs membres au Parlement Européen. Ainsi les partis LR (Les

\footnotetext{
${ }^{7}$ Le questionnaire n'a pas été envoyé aux membres du Bundesrat car cette chambre inclut seulement une minorité des représentants des gouvernements des Länder allemands.

${ }^{8}$ Voir l'Annexe B pour plus de détails sur la forme de l'enquête.
} 
Républicains) et CDU/CSU (Union chrétienne-démocrate d'Allemagne et Union chrétiennesociale de Bavière) sont membres du groupe PPE (Parti populaire européen); les partis membres du groupe S\&D (Alliance progressiste des socialistes et démocrates) sont le PS (Parti Socialiste), le PRG (Parti Radical de Gauche) et le SPD (Parti social-démocrate d'Allemagne). Le parti UDI (Union des Démocrates et Indépendants) est membre du groupe ALDE (Alliance des libéraux et des démocrates pour l'Europe); Bündnis 90/Die Grünen et EELV (Europe écologie-les Verts) sont membres du groupe des Verts/Alliance libre européenne; Die Linke, le groupe Gauche démocrate et républicaine et le PC (Parti Communiste) sont membres du groupe GUE/NGL (Gauche unitaire européenne/Gauche verte nordique) et le FN (Front national) est membre du groupe ENL (Europe des nations et des libertés). Vingt-trois parlementaires ne correspondent à aucun de ces groupes.

TABLE 1 - Participation par partis politiques

\begin{tabular}{l|cc|c}
\hline Parti & Réponse (\%) & Pas de réponse (\%) & Total \\
\hline PPE & $13,5 \%$ & $86,5 \%$ & 650 \\
S\&D & $17,9 \%$ & $82,1 \%$ & 616 \\
Autres & $11,9 \%$ & $88,1 \%$ & 286 \\
\hline Total & $14,9 \%$ & $85,1 \%$ & 1552 \\
\hline
\end{tabular}

La catégorie "Autres" est composée des membres affiliés aux groupes ALDE (total de 72), Verts/ALE (total de 90), GUE/NGL (total de 98) et ENL (total de 3), et non classifiés (23 au total). Étant donné leurs nombres ainsi que leurs taux de réponses, nous avons décidé de les grouper afin de préserver la confidentialité des réponses exprimées.

Certaines caractéristiques individuelles ont également été prises en compte : il s'agit de l'âge, du sexe, du nombre d'années au parlement, de l'obtention ou non du baccalauréat, de la poursuite ou non d'études supérieures (voir l'Annexe A pour plus de détails). Nous avons également des données sur leur participation à la Commission des Finances ou à celle des Affaires Européennes. Le nombre de réponses dans les différents groupes (voir Table 1) et notre engagement à garantir la confidentialité des réponses impliquent que nous présentons ici les résultats des parlementaires rattachés aux groupes PPE et S\&D.

La Table 1 ainsi que la Table 10 (dans l'Annexe A.2) mettent en avant de manière descriptive des taux de réponses différant selon la nationalité, l'appartenance politique et les caractéristiques individuelles mentionnées ci-dessus.

Dans la section 3.1 nous estimerons la décision de participation à l'enquête en fonction de ces caractéristiques et la section 4.1 s'attachera à contrôler pour d'éventuels effets de sélection.

Marché du travail et intégration européenne. Nous présentons dans cet article les réponses aux cinq questions relatives au marché du travail exposées dans l'introduction. 
Statistiques descriptives. Nous commençons par faire une analyse descriptive de la distribution des réponses par parti politique puis par nationalité.

La proposition d'introduire des directives contraignantes relatives au marché du travail des membres de l'UE trouve un fort appui dans le groupe S\&D, et peu de soutien dans le groupe PPE qui est en grande partie fortement contre, même si l'on en trouve une proportion non-négligeable qui l'appuie légèrement. Les questions concernant la politique salariale et la flexibilité du marché du travail présentent des opinions très divergentes avec les membres des partis $\mathrm{S} \& \mathrm{D}$ et $\mathrm{PPE}$ répartis de part et d'autre de la distribution des réponses. Cette divergence $\mathrm{S} \& D$ et PPE se retrouve dans les réponses à la question d'une assurance chômage européenne, avec une très forte concentration (près de 40\%) de membres du groupe PPE qui y sont très opposés. La proposition selon laquelle l'UE devrait jouer un rôle renforcé dans la politique d'immigration des États membres semble être la plus consensuelle même si environ $18 \%$ des membres du groupe PPE sont fortement contre.

FiguRE 1 - Distribution des réponses par parti politique (en pourcentage)
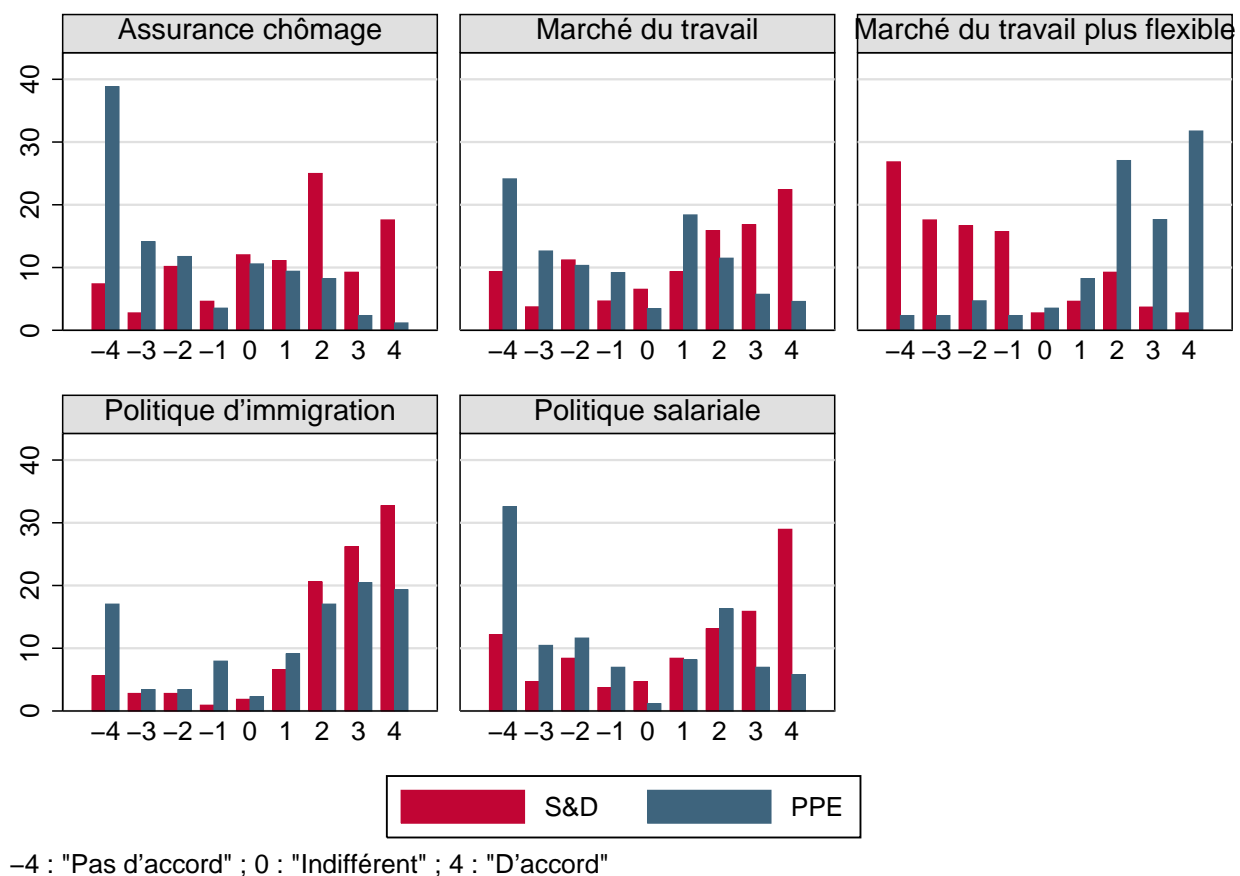

En considérant les réponses à travers le filtre de la nationalité nous observons que la proposition concernant le marché du travail semble assez clivante, les parlementaires allemands y étant plus opposés que les parlementaires français. Les proportions sont cependant similaires pour les parlementaires avec de faibles préférences dans un sens ou dans l'autre. La proposition selon laquelle les politiques salariales des États membres pourraient être davantage intégrées trouve un fort soutien de la part des parlementaires français, et une forte opposi- 
tion de la part des parlementaires allemands. La création d'une assurance chômage commune européenne rencontre une forte opposition de la part des parlementaires allemands. Les réponses aux questions relatives à un marché du travail plus flexible ainsi qu'à une politique d'immigration commune semblent montrer un certain consensus entre les parlementaires des deux pays.

Figure 2 - Distribution des réponses par nationalité (en pourcentage)
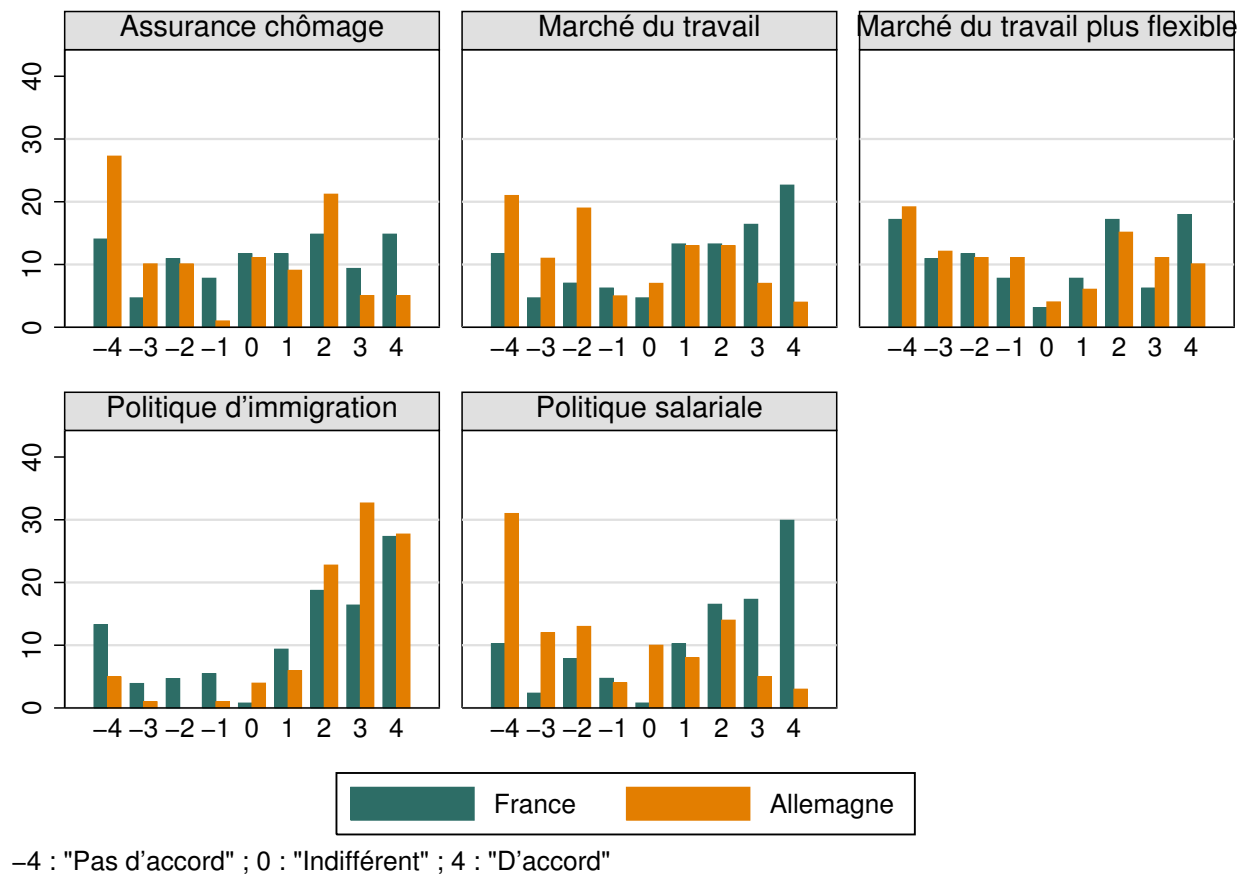

NB : Nous prenons ici en compte tous les parlementaires.

Selon les tests statistiques de Mann-Whitney et Chi2, les distributions des réponses françaises et allemandes concernant un marché du travail plus flexible ne sont pas statistiquement différentes. Les médianes pour les questions de l'immigration, d'une assurance chômage et de la flexibilité du marché du travail ne sont pas statistiquement différentes entre parlementaires français et allemands.

Les distributions en fonction de l'appartenance politique (S\&D ou PPE) ressortent toutes comme statistiquement différentes; les médianes également.

Il apparaîtrait que la question de l'immigration recevrait un support majoritaire de la part des parlementaires français et allemands. Les proportions de parlementaires en faveur d'une assurance chômage européenne et d'un marché du travail plus flexible ne sont statistiquement pas différentes de $50 \%$ (tests de proportions). Ces trois domaines pourraient donc représenter 
un terrain d'entente franco-allemand (voir l'Annexe B pour plus de détails).

\section{Analyse économétrique}

Le but de cette analyse économétrique n'est pas d'établir de lien de causalité entre une idéologie politique et/ou une nationalité et une opinion sur l'intégration européenne. Nous cherchons, tout en contrôlant par les caractéristiques individuelles des parlementaires, à mettre en lumière, dans le contexte actuel, des terrains d'entente entre les deux pays ainsi qu'à déterminer les domaines où les clivages idéologiques sont les moins élevés.

Nous avons commencé par comparer les différences statistiquement significatives entre les réponses données par les parlementaires français et allemands puis par les groupes S\&D et PPE. Afin d'analyser les données obtenues et de contrôler par des facteurs individuels, nous avons utilisé un modèle Probit ordonné.

\subsection{Déterminants de la participation}

Nous commençons par déterminer les facteurs de participation en estimant un modèle Probit binaire, où la variable dépendante Part est égale à 1 si le/la parlementaire a répondu, 0 si il/elle n'a pas répondu à l'enquête.

Nous utilisons les variables explicatives mentionnées dans la section 2 :

- $F r=1$ si le/la parlementaire est français(e), 0 si il/elle est allemand(e);

- $S \& D=1$ si le/la parlementaire appartient au groupe $S \& D, 0$ pour le groupe PPE.

- Femme = 1 si c'est une femme, 0 si c'est un homme ;

- Age l'âge du/de la parlementaire ;

- Annees le nombre d'années passées au parlement;

- $B a c=1$ si il/elle a obtenu son baccalauréat (Abitur en Allemagne);

- Etudes = 1 si il/elle a poursuivi des études supérieures.

Ces variables sont les composantes d'un vecteur $\boldsymbol{X}$.

Et nous estimons le modèle Probit suivant :

$\operatorname{Prob}\left(\right.$ Part $\left._{i}=1 \mid \boldsymbol{X}_{\boldsymbol{i}}\right)=\Phi\left(b_{1} F r_{i}+b_{2} S \& D_{i}+b_{3}\right.$ Femme $_{i}+b_{4}$ Age $_{i}+b_{5}$ Age $_{i}^{2}+b_{6}$ Annees $_{i}+b_{7}$ Bac $_{i}+b_{8}$ Etudes $\left._{i}\right)$. 
TABLE 2 - Déterminants de la participation : Effets marginaux moyens

\begin{tabular}{lc}
\hline & Participation \\
\hline Fr & -0.039 \\
S\&D & $(0.024)$ \\
& $0.051^{* *}$ \\
Femme & $(0.022)$ \\
Age & -0.033 \\
& $(0.023)$ \\
Annees & $0.0038^{* * *}$ \\
& $(0.0013)$ \\
Bac & $-0.0029^{*}$ \\
& $(0.0015)$ \\
Etudes & 0.061 \\
& $(0.043)$ \\
& -0.026 \\
Observations & $(0.051)$ \\
\hline Les écarts-types sont données entre parenthèses \\
\multirow{2}{*}{$* * * \mathrm{p}<0.01, * * \mathrm{p}<0.05, * \mathrm{p}<0.1$}
\end{tabular}

Les résultats présentés dans la Table 2 révèlent un effet positif et significatif à un niveau de $95 \%$ pour l'affiliation politique (effet marginal moyen de $5 \%$ : ceteris paribus, un/une parlementaire du groupe S\&D a en moyenne une probabilité de $5 \%$ supérieure à un/une parlementaire du groupe PPE d'avoir répondu à l'enquête) : les parlementaires membres du groupe S\&D ont donc proportionnellement plus répondu à notre enquête.

Pour les autres variables nous ne trouvons pas d'effet significatif ou un effet significatif mais très faible. En particulier, l'âge a un effet faiblement positif et significatif à un niveau de 99\% (effet marginal moyen de 0,4\%) et le nombre d'années passées au parlement a un effet faiblement négatif et significatif à un niveau de $90 \%$ (effet marginal moyen de 0,3\%).

Dans la section 4.1, nous prenons en compte les éventuels effets de sélection en utilisant un modèle de sélection de Heckman.

\subsection{Méthode d'estimation : Le modèle Probit ordonné}

Nous analysons maintenant les réponses obtenues.

Le modèle estimé est le suivant : soit $Y_{i}$ la variable indiquant la réponse à l'une des questions et $Y_{i}^{*}$ la variable latente associée à $Y_{i}$ telle que

$$
Y_{i}^{*}=\beta_{1} F r_{i}+\beta_{2} S D_{i}+\gamma \boldsymbol{Z}_{i}+\epsilon_{i}
$$

avec $\epsilon \sim \mathcal{N}(0,1), i \in\{1, \ldots, \mathrm{N}\}, \mathrm{N}$ étant le nombre de parlementaires ayant répondu à cette question donnée. 
Il existe $\alpha_{-5}, \alpha_{-3}, \ldots, \alpha_{4}\left(\alpha_{-5}=-\infty, \alpha_{4}=+\infty\right)$ tels que :

$$
\forall j \in\{-4, \ldots, 4\}, \quad Y_{i}=j \quad \Longleftrightarrow \quad \alpha_{j-1}<Y^{*}{ }_{i} \leq \alpha_{j} .
$$

D'où pour tout $j \in\{-4, \ldots, 4\}$,

$$
\operatorname{Prob}\left(Y_{i}=j \mid X_{i}\right)=\operatorname{Prob}\left(\alpha_{j-1}<Y_{i}^{*} \leq \alpha_{j} \mid X_{i}\right)
$$

ou encore, comme $\epsilon \sim \mathcal{N}(0,1)$,

$$
\operatorname{Prob}\left(Y_{i}=j \mid X_{i}\right)=\Phi\left(\alpha_{j}-\boldsymbol{X}_{\boldsymbol{i}}^{\boldsymbol{T}} \boldsymbol{\lambda}\right)-\Phi\left(\alpha_{j-1}-\boldsymbol{X}_{\boldsymbol{i}}^{\boldsymbol{T}} \boldsymbol{\lambda}\right)
$$

avec $\boldsymbol{X}_{\boldsymbol{i}}=\left(F r_{i}, S D_{i}, \boldsymbol{Z}_{i}\right)$ et $\boldsymbol{\lambda}=\left(\beta_{1}, \beta_{2}, \gamma\right)$.

Les composantes de $\boldsymbol{X}$ (variables explicatives) sont les mêmes que dans la section 3.1 :

\section{Variables principales :}

- $F r=1$ si le/la parlementaire est français(e), 0 si il/elle est allemand(e);

- $S \& D=1$ si le/la parlementaire appartient au groupe S\&D, 0 pour le groupe PPE.

\section{Variables de contrôle $(Z)$ :}

- Femme $=1$ si c'est une femme, 0 si c'est un homme ;

- Age l'âge du/de la parlementaire (nous ajoutons un terme quadratique pour cette variable);

- Annees le nombre d'années passées au parlement;

- $B a c=1$ si il/elle a obtenu son baccalauréat (Abitur en Allemagne);

- Etudes = 1 si il/elle a poursuivi des études supérieures.

Les coefficients $\beta_{1}, \beta_{2}$ et $\gamma_{1}, \gamma_{2}, \gamma_{3}, \gamma_{4}, \gamma_{5}$ et $\gamma_{6}$ ne pouvant que donner le signe de l'effet de la variable associée sur la variable dépendante, nous présentons plutôt les effets marginaux moyens $E_{j}^{k}, k \in\{1,2,3,4,6,7,8\}$ (moyenne des effets d'un changement marginal de $X^{k}$ la k-ième composante de $\boldsymbol{X}$ - sur la probabilité que $Y=j$ ) : 
Pour les variables discrètes (dichotomiques) $:^{9}$

$$
E_{j}^{k}=\frac{1}{N} \sum_{i=1}^{N} \Phi\left(\alpha_{j}-\boldsymbol{X}_{i}^{-k T} \boldsymbol{\lambda}^{-k}-\lambda^{k}\right)-\Phi\left(\alpha_{j-1}-\boldsymbol{X}_{\boldsymbol{i}}^{-k \boldsymbol{T}} \boldsymbol{\lambda}^{-\boldsymbol{k}}\right)
$$

Pour les variables continues :

$$
E_{j}^{k}=\frac{1}{N} \sum_{i=1}^{N} \frac{\partial \operatorname{Prob}\left(Y_{i}=j \mid \boldsymbol{X}_{\boldsymbol{i}}\right)}{\partial X_{i}^{k}}=\frac{\lambda^{k}}{N} \sum_{i=1}^{N} \phi\left(\alpha_{j-1}-\boldsymbol{X}_{\boldsymbol{i}}^{\boldsymbol{T}} \boldsymbol{\lambda}\right)-\phi\left(\alpha_{j}-\boldsymbol{X}_{\boldsymbol{i}}^{\boldsymbol{T}} \boldsymbol{\lambda}\right) .
$$

En raison du terme quadratique, la formule diffère pour la variable Age :

$$
E_{j}^{4}=\frac{1}{N} \sum_{i=1}^{N}\left(\lambda^{4}+2 \lambda^{5} A g e_{i}\right)\left(\phi\left(\alpha_{j-1}-\boldsymbol{X}_{\boldsymbol{i}}^{\boldsymbol{T}} \boldsymbol{\lambda}\right)-\phi\left(\alpha_{j}-\boldsymbol{X}_{\boldsymbol{i}}^{\boldsymbol{T}} \boldsymbol{\lambda}\right)\right) .
$$

\subsection{Résultats des estimations}

Nous présentons maintenant les résultats des différentes questions.

\section{Marché du travail : "L'UE pourrait donner des directives contraignantes aux} États membres relatives au marché du travail". Nous trouvons un effet significatif (à un niveau de 99\%) pour la nationalité et l'appartenance politique. Les deux effets sont positifs : la probabilité d'être plus en faveur de la proposition indiquée ceteris paribus augmente avec le fait d'être français d'une part et d'appartenir au groupe S\&D d'autre part. Les effets sont du même ordre de grandeur pour les deux variables. Le nombre d'années passées au parlement a également un léger effet négatif et significatif à un niveau de $95 \%$.

Les effets marginaux moyens pour chaque réponse possible sont donnés dans le tableau et le graphe ci-dessous $:^{10}$

\footnotetext{
${ }^{9} \boldsymbol{u}^{-\boldsymbol{k}}$ représente le vecteur $\boldsymbol{u}$ privé de sa k-ième composante.

${ }^{10}$ Nous pouvons par exemple interpréter le premier chiffre dans la table de la façon suivante : ceteris paribus, en moyenne, la probabilité qu'un/une parlementaire français(e) ne soit pas d'accord avec cette proposition (coche la case "-4") est de $15 \%$ plus basse que pour un/une parlementaire allemand(e).
} 
TABLE 3 - Marché du travail : Effets marginaux moyens

\begin{tabular}{|c|c|c|c|c|c|c|c|c|c|}
\hline & $(-4)$ & $(-3)$ & $(-2)$ & $(-1)$ & $(0)$ & (1) & (2) & (3) & (4) \\
\hline $\mathrm{Fr}$ & $\begin{array}{c}-0.15^{* * *} \\
(0.040)\end{array}$ & $\begin{array}{c}-0.039^{* * *} \\
(0.014)\end{array}$ & $\begin{array}{c}-0.040 * * * \\
(0.014)\end{array}$ & $\begin{array}{c}-0.014^{* *} \\
(0.0060)\end{array}$ & $\begin{array}{c}-0.0066^{*} \\
(0.0038)\end{array}$ & $\begin{array}{c}0.0069 \\
(0.0082)\end{array}$ & $\begin{array}{c}0.042^{* * *} \\
(0.016)\end{array}$ & $\begin{array}{c}0.072^{* * *} \\
(0.023)\end{array}$ & $\begin{array}{l}0.13^{* * *} \\
(0.031)\end{array}$ \\
\hline$S \& D$ & $\begin{array}{c}-0.14^{* * *} \\
(0.038)\end{array}$ & $\begin{array}{c}-0.038^{* * *} \\
(0.014)\end{array}$ & $\begin{array}{c}-0.040^{* * *} \\
(0.014)\end{array}$ & $\begin{array}{c}-0.014^{* *} \\
(0.0060)\end{array}$ & $\begin{array}{c}-0.0073^{*} \\
(0.0039)\end{array}$ & $\begin{array}{c}0.0044 \\
(0.0078)\end{array}$ & $\begin{array}{c}0.040^{* * *} \\
(0.015)\end{array}$ & $\begin{array}{c}0.071^{* * *} \\
(0.022)\end{array}$ & $\begin{array}{c}0.13^{* * *} \\
(0.032)\end{array}$ \\
\hline Femme & $\begin{array}{c}-0.0049 \\
(0.040)\end{array}$ & $\begin{array}{l}-0.0012 \\
(0.0098)\end{array}$ & $\begin{array}{c}-0.0013 \\
(0.011)\end{array}$ & $\begin{array}{l}-0.00047 \\
(0.0040)\end{array}$ & $\begin{array}{c}-0.00027 \\
(0.0023)\end{array}$ & $\begin{array}{l}-0.000022 \\
(0.00039)\end{array}$ & $\begin{array}{c}0.0011 \\
(0.0087)\end{array}$ & $\begin{array}{l}0.0022 \\
(0.018)\end{array}$ & $\begin{array}{l}0.0049 \\
(0.041)\end{array}$ \\
\hline Age & $\begin{array}{c}-0.0030 * \\
(0.0018)\end{array}$ & $\begin{array}{l}-0.00076 \\
(0.00050)\end{array}$ & $\begin{array}{l}-0.00082 \\
(0.00055)\end{array}$ & $\begin{array}{l}-0.00032 \\
(0.00024)\end{array}$ & $\begin{array}{l}-0.00019 \\
(0.00015)\end{array}$ & $\begin{array}{c}-0.000064 \\
(0.00019)\end{array}$ & $\begin{array}{l}0.00063^{*} \\
(0.00038)\end{array}$ & $\begin{array}{c}0.0014 \\
(0.00083)\end{array}$ & $\begin{array}{c}0.0032 \\
(0.0021)\end{array}$ \\
\hline Annees & $\begin{array}{c}0.0059 * * \\
(0.0023)\end{array}$ & $\begin{array}{l}0.0014^{* *} \\
(0.00065)\end{array}$ & $\begin{array}{l}0.0015^{* *} \\
(0.00067)\end{array}$ & $\begin{array}{l}0.00056^{*} \\
(0.00029)\end{array}$ & $\begin{array}{l}0.00031^{*} \\
(0.00018)\end{array}$ & $\begin{array}{c}5.8 \mathrm{e}-06 \\
(0.00027)\end{array}$ & $\begin{array}{r}-0.0013^{* *} \\
(0.00059)\end{array}$ & $\begin{array}{r}-0.0026^{* *} \\
(0.0011)\end{array}$ & $\begin{array}{c}-0.0058^{* *} \\
(0.0023)\end{array}$ \\
\hline $\mathrm{Bac}$ & $\begin{array}{c}0.082 \\
(0.064)\end{array}$ & $\begin{array}{c}0.022 \\
(0.020)\end{array}$ & $\begin{array}{c}0.026 \\
(0.025)\end{array}$ & $\begin{array}{c}0.011 \\
(0.012)\end{array}$ & $\begin{array}{c}0.0074 \\
(0.0092)\end{array}$ & $\begin{array}{l}0.0081 \\
(0.015)\end{array}$ & $\begin{array}{c}-0.012^{* *} \\
(0.0053)\end{array}$ & $\begin{array}{l}-0.036 \\
(0.026)\end{array}$ & $\begin{array}{l}-0.11 \\
(0.12)\end{array}$ \\
\hline Etudes & $\begin{array}{l}-0.16 \\
(0.11)\end{array}$ & $\begin{array}{c}-0.030^{*} \\
(0.017)\end{array}$ & $\begin{array}{c}-0.026^{* *} \\
(0.011)\end{array}$ & $\begin{array}{c}-0.0066^{*} \\
(0.0034)\end{array}$ & $\begin{array}{l}-0.0012 \\
(0.0039)\end{array}$ & $\begin{array}{c}0.016 \\
(0.019)\end{array}$ & $\begin{array}{c}0.042 \\
(0.031)\end{array}$ & $\begin{array}{l}0.063^{*} \\
(0.037)\end{array}$ & $\begin{array}{l}0.11^{* *} \\
(0.050)\end{array}$ \\
\hline Observations & 183 & 183 & 183 & 183 & 183 & 183 & 183 & 183 & 183 \\
\hline
\end{tabular}

Figure 3 - Marché du travail : Effets marginaux moyens avec intervalles de confiance à $95 \%$

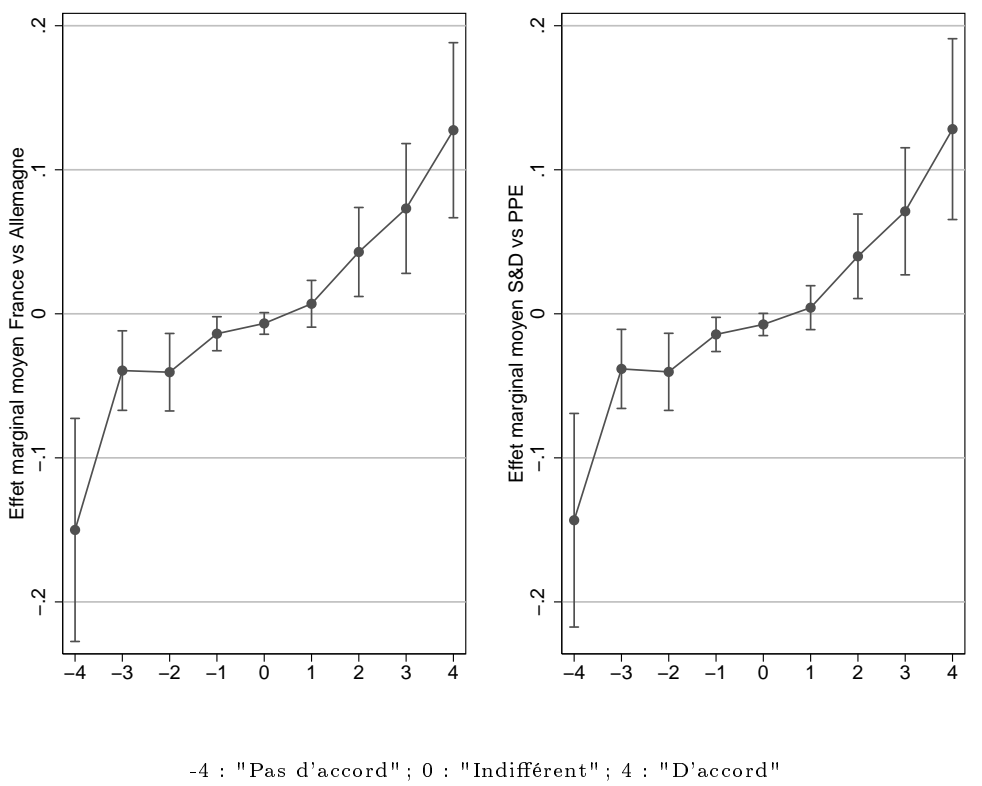

2. Politique salariale : "l'UE pourrait avoir des droits d'intervention plus importants dans les politiques salariales des États membres". Nous trouvons un effet significatif (à un niveau de 99\%) pour la nationalité et l'appartenance politique. Les deux effets sont positifs : la probabilité d'être plus en faveur de la proposition indiquée ceteris paribus augmente avec le fait d'être français d'une part et d'appartenir au groupe S\&D d'autre 
part. Les effets ne sont pas du même ordre de grandeur : la nationalité a un plus fort effet que l'affiliation politique.

L'âge (respectivement le nombre d'années au parlement) a un faible effet positif (resp. négatif) significatif à un niveau de 95\% (resp. 99\%). En ce qui concerne les études, avoir le baccalauréat (resp. avoir poursuivi des études supérieures) diminue (resp. augmente) la probabilité d'être plus en faveur de cette proposition. Ces facteurs ont tous deux un effet significatif à un niveau de $95 \%$.

Les effets marginaux moyens pour chaque réponse possible sont donnés dans le tableau et le graphe ci-dessous :

TABLE 4 - Politique salariale : Effets marginaux moyens

\begin{tabular}{|c|c|c|c|c|c|c|c|c|c|}
\hline & $(-4)$ & $(-3)$ & $(-2)$ & $(-1)$ & $(0)$ & $(1)$ & $(2)$ & $(3)$ & (4) \\
\hline $\mathrm{Fr}$ & $\begin{array}{c}-0.24 * * * \\
(0.045)\end{array}$ & $\begin{array}{c}-0.055^{* * *} \\
(0.017)\end{array}$ & $\begin{array}{c}-0.046^{* * *} \\
(0.014)\end{array}$ & $\begin{array}{c}-0.012^{* *} \\
(0.0061)\end{array}$ & $\begin{array}{l}-0.0032 \\
(0.0031)\end{array}$ & $\begin{array}{c}0.0053 \\
(0.0072)\end{array}$ & $\begin{array}{c}0.052^{* * *} \\
(0.018)\end{array}$ & $\begin{array}{c}0.097^{* * * *} \\
(0.025)\end{array}$ & $\begin{array}{c}0.20 * * * \\
(0.035)\end{array}$ \\
\hline S\&D & $\begin{array}{c}-0.17^{* * *} \\
(0.040)\end{array}$ & $\begin{array}{c}-0.037^{* * *} \\
(0.012)\end{array}$ & $\begin{array}{c}-0.032^{* * *} \\
(0.011)\end{array}$ & $\begin{array}{c}-0.0099^{* *} \\
(0.0045)\end{array}$ & $\begin{array}{l}-0.0033 \\
(0.0023)\end{array}$ & $\begin{array}{c}0.000013 \\
(0.0045)\end{array}$ & $\begin{array}{c}0.028 * * \\
(0.012)\end{array}$ & $\begin{array}{c}0.064^{* * *} \\
(0.020)\end{array}$ & $\begin{array}{c}0.16^{* * *} \\
(0.034)\end{array}$ \\
\hline Femme & $\begin{array}{c}0.00037 \\
(0.042)\end{array}$ & $\begin{array}{c}0.000071 \\
(0.0081)\end{array}$ & $\begin{array}{c}0.000066 \\
(0.0075)\end{array}$ & $\begin{array}{c}0.000022 \\
(0.0025)\end{array}$ & $\begin{array}{c}8.8 \mathrm{e}-06 \\
(0.00099)\end{array}$ & $\begin{array}{c}6.8 \mathrm{e}-06 \\
(0.00077)\end{array}$ & $\begin{array}{c}-0.000042 \\
(0.0047)\end{array}$ & $\begin{array}{c}-0.00012 \\
(0.013)\end{array}$ & $\begin{array}{c}-0.00039 \\
(0.044)\end{array}$ \\
\hline Age & $\begin{array}{c}-0.0047^{* * *} \\
(0.0017)\end{array}$ & $\begin{array}{c}-0.0010^{* *} \\
(0.00048)\end{array}$ & $\begin{array}{c}-0.0010^{* *} \\
(0.00048)\end{array}$ & $\begin{array}{c}-0.00040^{*} \\
(0.00021)\end{array}$ & $\begin{array}{l}-0.00018 \\
(0.00011)\end{array}$ & $\begin{array}{l}-0.00025 \\
(0.00018)\end{array}$ & $\begin{array}{c}0.00030 \\
(0.00027)\end{array}$ & $\begin{array}{c}0.0014^{* *} \\
(0.00058)\end{array}$ & $\begin{array}{c}0.0059^{* *} \\
(0.0024)\end{array}$ \\
\hline Annees & $\begin{array}{c}0.0074^{* * *} \\
(0.0024)\end{array}$ & $\begin{array}{l}0.0014^{* *} \\
(0.00057)\end{array}$ & $\begin{array}{l}0.0013^{* *} \\
(0.00053)\end{array}$ & $\begin{array}{c}0.00045^{* *} \\
(0.00022)\end{array}$ & $\begin{array}{c}0.00018 \\
(0.00011)\end{array}$ & $\begin{array}{c}0.00014 \\
(0.00017)\end{array}$ & $\begin{array}{c}-0.00083^{* *} \\
(0.00042)\end{array}$ & $\begin{array}{c}-0.0023^{* * *} \\
(0.00088)\end{array}$ & $\begin{array}{c}-0.0078^{* * *} \\
(0.0025)\end{array}$ \\
\hline $\mathrm{Bac}$ & $\begin{array}{c}0.16^{* * *} \\
(0.055)\end{array}$ & $\begin{array}{c}0.037 * * \\
(0.017)\end{array}$ & $\begin{array}{l}0.040^{*} \\
(0.021)\end{array}$ & $\begin{array}{c}0.017 \\
(0.011)\end{array}$ & $\begin{array}{c}0.0088 \\
(0.0066)\end{array}$ & $\begin{array}{c}0.017 \\
(0.014)\end{array}$ & $\begin{array}{l}0.0078 \\
(0.019)\end{array}$ & $\begin{array}{c}-0.029 * * \\
(0.012)\end{array}$ & $\begin{array}{l}-0.26^{*} \\
(0.14)\end{array}$ \\
\hline Etudes & $\begin{array}{c}-0.27^{* *} \\
(0.13)\end{array}$ & $\begin{array}{c}-0.030^{* * *} \\
(0.010)\end{array}$ & $\begin{array}{c}-0.017^{* *} \\
(0.0083)\end{array}$ & $\begin{array}{c}0.000085 \\
(0.0070)\end{array}$ & $\begin{array}{c}0.0029 \\
(0.0051)\end{array}$ & $\begin{array}{c}0.015 \\
(0.015)\end{array}$ & $\begin{array}{l}0.052^{*} \\
(0.031)\end{array}$ & $\begin{array}{c}0.078 * * \\
(0.033)\end{array}$ & $\begin{array}{c}0.17^{* * *} \\
(0.051)\end{array}$ \\
\hline Observations & 182 & 182 & 182 & 182 & 182 & 182 & 182 & 182 & 182 \\
\hline
\end{tabular}


FIGURE 4 - Politique salariale : Effets marginaux moyens avec intervalles de confiance à $95 \%$
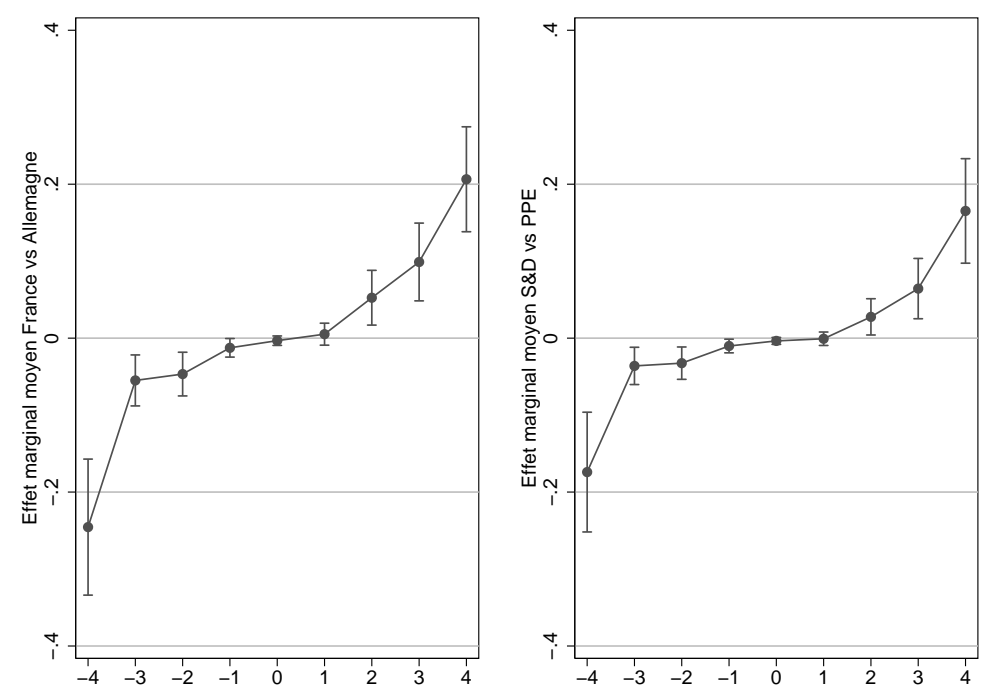

-4 : "Pas d'accord" ; 0 : "Indifférent"; 4 : "D'accord"

\section{Politique d'immigration : "L'UE devrait jouer un rôle renforcé dans la poli-} tique d'immigration des États membres". Nous trouvons un effet significatif (à un niveau de 99\%) pour la nationalité et l'appartenance politique. Les deux effets sont de sens contraires : être français diminue la probabilité d'être plus en faveur de la proposition, ceteris paribus et appartenir au groupe $\mathrm{S} \& \mathrm{D}$ augmente la probabilité d'être plus en faveur de la proposition indiquée ceteris paribus.

Les effets marginaux moyens pour chaque réponse possible sont donnés dans le tableau et le graphe ci-dessous : 
TABle 5 - Politique d'immigration : Effets marginaux moyens

\begin{tabular}{|c|c|c|c|c|c|c|c|c|c|}
\hline & $(-4)$ & $(-3)$ & $(-2)$ & $(-1)$ & (0) & (1) & (2) & (3) & (4) \\
\hline $\mathrm{Fr}$ & $\begin{array}{c}0.076^{* * *} \\
(0.029)\end{array}$ & $\begin{array}{c}0.016^{*} \\
(0.0083)\end{array}$ & $\begin{array}{c}0.012 * \\
(0.0067)\end{array}$ & $\begin{array}{c}0.013^{*} \\
(0.0072)\end{array}$ & $\begin{array}{c}0.0080 \\
(0.0050)\end{array}$ & $\begin{array}{c}0.024^{* *} \\
(0.011)\end{array}$ & $\begin{array}{l}0.023^{*} \\
(0.012)\end{array}$ & $\begin{array}{c}-0.029^{* *} \\
(0.013)\end{array}$ & $\begin{array}{c}-0.14^{* * *} \\
(0.054)\end{array}$ \\
\hline$S \& D$ & $\begin{array}{c}-0.11^{* * *} \\
(0.034)\end{array}$ & $\begin{array}{c}-0.023^{* *} \\
(0.011)\end{array}$ & $\begin{array}{c}-0.017^{* *} \\
(0.0085)\end{array}$ & $\begin{array}{c}-0.018^{* *} \\
(0.0088)\end{array}$ & $\begin{array}{l}-0.011^{*} \\
(0.0062)\end{array}$ & $\begin{array}{c}-0.032^{* * *} \\
(0.012)\end{array}$ & $\begin{array}{c}-0.026^{* *} \\
(0.012)\end{array}$ & $\begin{array}{c}0.050^{* * *} \\
(0.019)\end{array}$ & $\begin{array}{c}0.19^{* * *} \\
(0.049)\end{array}$ \\
\hline Femme & $\begin{array}{l}-0.020 \\
(0.033)\end{array}$ & $\begin{array}{l}-0.0040 \\
(0.0071)\end{array}$ & $\begin{array}{l}-0.0030 \\
(0.0054)\end{array}$ & $\begin{array}{l}-0.0033 \\
(0.0060)\end{array}$ & $\begin{array}{l}-0.0020 \\
(0.0037)\end{array}$ & $\begin{array}{c}-0.0060 \\
(0.011)\end{array}$ & $\begin{array}{c}-0.0058 \\
(0.011)\end{array}$ & $\begin{array}{l}0.0074 \\
(0.012)\end{array}$ & $\begin{array}{c}0.037 \\
(0.065)\end{array}$ \\
\hline Age & $\begin{array}{c}0.0024 \\
(0.0021)\end{array}$ & $\begin{array}{c}0.00039 \\
(0.00035)\end{array}$ & $\begin{array}{c}0.00028 \\
(0.00026)\end{array}$ & $\begin{array}{c}0.00029 \\
(0.00027)\end{array}$ & $\begin{array}{c}0.00017 \\
(0.00016)\end{array}$ & $\begin{array}{c}0.00044 \\
(0.00039)\end{array}$ & $\begin{array}{c}0.00015 \\
(0.00031)\end{array}$ & $\begin{array}{c}-0.0011 \\
(0.00093)\end{array}$ & $\begin{array}{l}-0.0030 \\
(0.0026)\end{array}$ \\
\hline Annees & $\begin{array}{c}-0.0014 \\
(0.0020)\end{array}$ & $\begin{array}{l}-0.00028 \\
(0.00040)\end{array}$ & $\begin{array}{l}-0.00021 \\
(0.00030)\end{array}$ & $\begin{array}{l}-0.00023 \\
(0.00033)\end{array}$ & $\begin{array}{l}-0.00014 \\
(0.00020)\end{array}$ & $\begin{array}{l}-0.00040 \\
(0.00057)\end{array}$ & $\begin{array}{l}-0.00036 \\
(0.00051)\end{array}$ & $\begin{array}{c}0.00055 \\
(0.00078)\end{array}$ & $\begin{array}{c}0.0025 \\
(0.0035)\end{array}$ \\
\hline $\mathrm{Bac}$ & $\begin{array}{l}-0.0057 \\
(0.079)\end{array}$ & $\begin{array}{r}-0.0011 \\
(0.015)\end{array}$ & $\begin{array}{c}-0.00081 \\
(0.011)\end{array}$ & $\begin{array}{c}-0.00089 \\
(0.012)\end{array}$ & $\begin{array}{c}-0.00054 \\
(0.0073)\end{array}$ & $\begin{array}{c}-0.0016 \\
(0.021)\end{array}$ & $\begin{array}{c}-0.0013 \\
(0.018)\end{array}$ & $\begin{array}{l}0.0022 \\
(0.032)\end{array}$ & $\begin{array}{c}0.0097 \\
(0.13)\end{array}$ \\
\hline Etudes & $\begin{array}{l}0.0011 \\
(0.069)\end{array}$ & $\begin{array}{c}0.00020 \\
(0.013)\end{array}$ & $\begin{array}{l}0.00015 \\
(0.0100)\end{array}$ & $\begin{array}{c}0.00017 \\
(0.011)\end{array}$ & $\begin{array}{l}0.00010 \\
(0.0066)\end{array}$ & $\begin{array}{c}0.00030 \\
(0.019)\end{array}$ & $\begin{array}{c}0.00026 \\
(0.017)\end{array}$ & $\begin{array}{c}-0.00041 \\
(0.026)\end{array}$ & $\begin{array}{c}-0.0018 \\
(0.12)\end{array}$ \\
\hline Observations & 185 & 185 & 185 & 185 & 185 & 185 & 185 & 185 & 185 \\
\hline
\end{tabular}

FiguRE 5 - Politique d'immigration : Effets marginaux moyens avec intervalles de confiance à $95 \%$

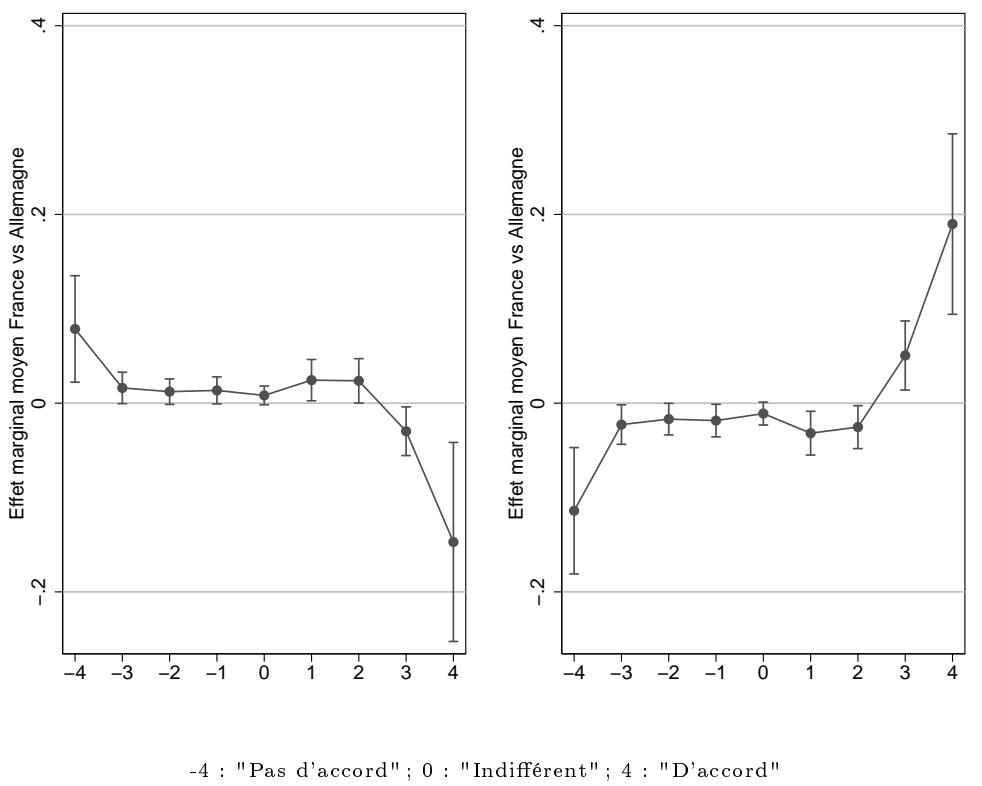

4. Assurance chômage européenne : "Pour pallier une éventuelle récession de certains États membres dans la zone euro il faudrait créer une assurance chômage commune européenne". Nous trouvons un effet significatif pour la nationalité (à un niveau de 95\%) et l'appartenance politique (à un niveau de 99\%). Les deux effets sont positifs : la probabilité d'être plus en faveur de la proposition indiquée augmente ceteris 
paribus avec le fait d'être français d'une part et d'appartenir au groupe S\&D d'autre part. Les effets ne sont pas du même ordre de grandeur : le clivage politique est beaucoup plus fort que la nationalité. De plus, lorsque nous faisons un Probit binaire nous ne trouvons pas d'effet significatif pour la nationalité (voir la section 4.1 et Annexe B).

Les effets marginaux moyens pour chaque réponse possible sont donnés dans le tableau et le graphe ci-dessous :

TABLE 6 - Assurance chômage européenne : Effets marginaux moyens

\begin{tabular}{|c|c|c|c|c|c|c|c|c|c|}
\hline & $(-4)$ & $(-3)$ & $(-2)$ & $(-1)$ & $(0)$ & $(1)$ & $(2)$ & (3) & (4) \\
\hline $\mathrm{Fr}$ & $\begin{array}{c}-0.097 * * \\
(0.042)\end{array}$ & $\begin{array}{c}-0.019 * * \\
(0.0096)\end{array}$ & $\begin{array}{c}-0.014^{* *} \\
(0.0071)\end{array}$ & $\begin{array}{l}-0.0024 \\
(0.0016)\end{array}$ & $\begin{array}{l}0.00033 \\
(0.0027)\end{array}$ & $\begin{array}{c}0.0085 \\
(0.0054)\end{array}$ & $\begin{array}{c}0.041^{* *} \\
(0.020)\end{array}$ & $\begin{array}{l}0.023^{*} \\
(0.012)\end{array}$ & $\begin{array}{c}0.060 * * \\
(0.025)\end{array}$ \\
\hline$S \& D$ & $\begin{array}{c}-0.30^{* * * *} \\
(0.050)\end{array}$ & $\begin{array}{c}-0.069^{* * *} \\
(0.020)\end{array}$ & $\begin{array}{c}-0.048^{* * *} \\
(0.016)\end{array}$ & $\begin{array}{l}-0.0062 \\
(0.0051)\end{array}$ & $\begin{array}{c}0.010 \\
(0.011)\end{array}$ & $\begin{array}{c}0.040^{* * *} \\
(0.014)\end{array}$ & $\begin{array}{c}0.15^{* * *} \\
(0.032)\end{array}$ & $\begin{array}{c}0.073^{* * *} \\
(0.022)\end{array}$ & $\begin{array}{c}0.15^{* * *} \\
(0.032)\end{array}$ \\
\hline Femme & $\begin{array}{c}0.036 \\
(0.050)\end{array}$ & $\begin{array}{c}0.0065 \\
(0.0085)\end{array}$ & $\begin{array}{c}0.0049 \\
(0.0061)\end{array}$ & $\begin{array}{l}0.00086 \\
(0.0010)\end{array}$ & $\begin{array}{c}-0.000030 \\
(0.0011)\end{array}$ & $\begin{array}{c}-0.0028 \\
(0.0044)\end{array}$ & $\begin{array}{l}-0.014 \\
(0.020)\end{array}$ & $\begin{array}{c}-0.0084 \\
(0.011)\end{array}$ & $\begin{array}{l}-0.023 \\
(0.030)\end{array}$ \\
\hline Age & $\begin{array}{l}-0.0026 \\
(0.0021)\end{array}$ & $\begin{array}{c}-0.00054 \\
(0.00050)\end{array}$ & $\begin{array}{l}-0.00043 \\
(0.00041)\end{array}$ & $\begin{array}{l}-0.000088 \\
(0.000098)\end{array}$ & $\begin{array}{r}-0.000049 \\
(0.00010)\end{array}$ & $\begin{array}{c}0.00017 \\
(0.00014)\end{array}$ & $\begin{array}{c}0.00100 \\
(0.00084)\end{array}$ & $\begin{array}{c}0.00063 \\
(0.00056)\end{array}$ & $\begin{array}{c}0.0019 \\
(0.0017)\end{array}$ \\
\hline Annees & $\begin{array}{c}0.0017 \\
(0.0027)\end{array}$ & $\begin{array}{c}0.00033 \\
(0.00053)\end{array}$ & $\begin{array}{c}0.00026 \\
(0.00041)\end{array}$ & $\begin{array}{c}0.000050 \\
(0.000082)\end{array}$ & $\begin{array}{c}0.000016 \\
(0.000051)\end{array}$ & $\begin{array}{l}-0.00012 \\
(0.00020)\end{array}$ & $\begin{array}{c}-0.00068 \\
(0.0011)\end{array}$ & $\begin{array}{l}-0.00041 \\
(0.00065)\end{array}$ & $\begin{array}{l}-0.0012 \\
(0.0018)\end{array}$ \\
\hline $\mathrm{Bac}$ & $\begin{array}{c}0.12 \\
(0.080)\end{array}$ & $\begin{array}{c}0.028 \\
(0.024)\end{array}$ & $\begin{array}{c}0.025 \\
(0.025)\end{array}$ & $\begin{array}{c}0.0064 \\
(0.0075)\end{array}$ & $\begin{array}{l}0.0081 \\
(0.013)\end{array}$ & $\begin{array}{l}-0.0020 \\
(0.0061)\end{array}$ & $\begin{array}{c}-0.039^{*} \\
(0.020)\end{array}$ & $\begin{array}{l}-0.030 \\
(0.022)\end{array}$ & $\begin{array}{l}-0.11 \\
(0.11)\end{array}$ \\
\hline Etudes & $\begin{array}{l}-0.16 \\
(0.13)\end{array}$ & $\begin{array}{c}-0.023^{*} \\
(0.013)\end{array}$ & $\begin{array}{c}-0.013 * * \\
(0.0053)\end{array}$ & $\begin{array}{c}-0.00044 \\
(0.0029)\end{array}$ & $\begin{array}{l}0.0075 \\
(0.012)\end{array}$ & $\begin{array}{c}0.018 \\
(0.018)\end{array}$ & $\begin{array}{c}0.064 \\
(0.048)\end{array}$ & $\begin{array}{c}0.032 \\
(0.022)\end{array}$ & $\begin{array}{l}0.077^{*} \\
(0.044)\end{array}$ \\
\hline Observations & 182 & 182 & 182 & 182 & 182 & 182 & 182 & 182 & 182 \\
\hline
\end{tabular}


FIgURE 6 - Assurance chômage européenne : Effets marginaux moyens avec intervalles de confiance à $95 \%$
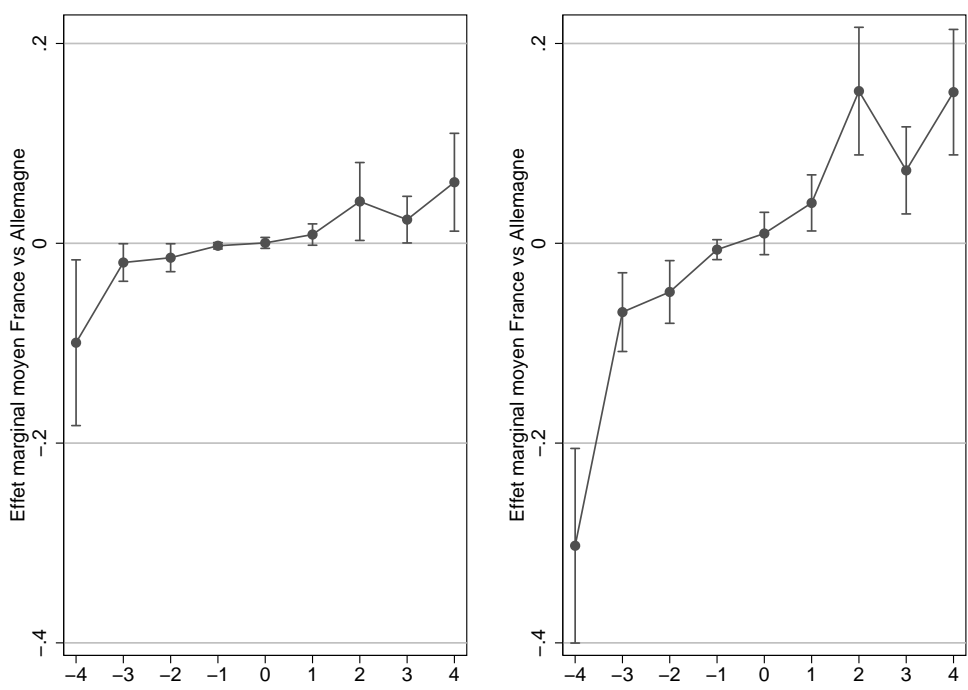

-4 : "Pas d'accord" ; 0 : "Indifférent" ; 4 : "D’accord"

5. Marché du travail plus flexible : "Une croissance plus forte dans la zone euro requière que les États comptabilisant un nombre important de chômeurs de longue durée rendent plus flexible leur marché du travail". Cette question révèle un clivage exclusivement partisan : le seul effet significatif (à un niveau de 99\%) se retrouve dans l'appartenance politique. Les effets sont très importants par rapport questions précédentes : la probabilité d'être plus en faveur de la proposition indiquée ceteris paribus augmente fortement avec le fait d'appartenir au groupe PPE.

Les effets marginaux moyens pour chaque réponse possible sont donnés dans le tableau et le graphe ci-dessous : 
TABLE 7 - Marché du travail plus flexible : Effets marginaux moyens

\begin{tabular}{|c|c|c|c|c|c|c|c|c|c|}
\hline & $(-4)$ & $(-3)$ & $(-2)$ & $(-1)$ & $(0)$ & (1) & $(2)$ & (3) & $(4)$ \\
\hline \multirow[t]{2}{*}{ Fr } & 0.00075 & 0.00023 & 0.00012 & 0.000024 & $-5.8 \mathrm{e}-06$ & -0.000027 & -0.00019 & -0.00021 & -0.00069 \\
\hline & $(0.033)$ & $(0.010)$ & $(0.0052)$ & $(0.0011)$ & $(0.00026)$ & $(0.0012)$ & $(0.0084)$ & $(0.0095)$ & $(0.031)$ \\
\hline \multirow[t]{2}{*}{$S \& D$} & $0.25^{* * *}$ & $0.14^{* * *}$ & $0.11^{* * *}$ & $0.054 * * *$ & 0.0072 & -0.0034 & $-0.12^{* * *}$ & $-0.14 * * *$ & $-0.29 * * *$ \\
\hline & $(0.040)$ & $(0.031)$ & $(0.026)$ & $(0.019)$ & $(0.0062)$ & $(0.0098)$ & $(0.031)$ & $(0.033)$ & $(0.048)$ \\
\hline \multirow[t]{2}{*}{ Femme } & -0.0029 & -0.00089 & -0.00045 & -0.000095 & 0.000022 & 0.00010 & 0.00072 & 0.00081 & 0.0026 \\
\hline & $(0.040)$ & $(0.012)$ & $(0.0063)$ & $(0.0013)$ & $(0.00029)$ & $(0.0014)$ & $(0.010)$ & $(0.011)$ & $(0.037)$ \\
\hline \multirow[t]{2}{*}{ Age } & -0.0012 & -0.00046 & -0.00030 & -0.00016 & -0.000034 & -0.000036 & 0.00013 & 0.00034 & 0.0018 \\
\hline & $(0.0017)$ & $(0.00062)$ & $(0.00037)$ & $(0.00016)$ & $(0.000032)$ & $(0.000053)$ & $(0.00037)$ & $(0.00048)$ & $(0.0020)$ \\
\hline \multirow[t]{2}{*}{ Annees } & -0.0011 & -0.00034 & -0.00017 & -0.000036 & $8.5 \mathrm{e}-06$ & 0.000040 & 0.00028 & 0.00031 & 0.0010 \\
\hline & $(0.0023)$ & $(0.00071)$ & $(0.00036)$ & $(0.000083)$ & $(0.000020)$ & $(0.000084)$ & $(0.00058)$ & $(0.00066)$ & $(0.0021)$ \\
\hline \multirow[t]{2}{*}{$\mathrm{Bac}$} & 0.036 & 0.012 & 0.0066 & 0.0019 & -0.000063 & -0.00098 & -0.0086 & -0.010 & -0.036 \\
\hline & $(0.080)$ & $(0.030)$ & $(0.018)$ & $(0.0064)$ & $(0.00051)$ & $(0.0016)$ & $(0.018)$ & $(0.023)$ & $(0.091)$ \\
\hline \multirow[t]{2}{*}{ Etudes } & -0.074 & -0.019 & -0.0078 & -0.00031 & 0.0011 & 0.0033 & 0.019 & 0.020 & 0.057 \\
\hline & $(0.094)$ & $(0.019)$ & $(0.0071)$ & $(0.0025)$ & $(0.0019)$ & $(0.0049)$ & $(0.025)$ & $(0.024)$ & $(0.062)$ \\
\hline Observations & 182 & 182 & 182 & 182 & 182 & 182 & 182 & 182 & 182 \\
\hline
\end{tabular}

Figure 7 - Marché du travail plus flexible : Effets marginaux moyens avec intervalles de confiance à $95 \%$

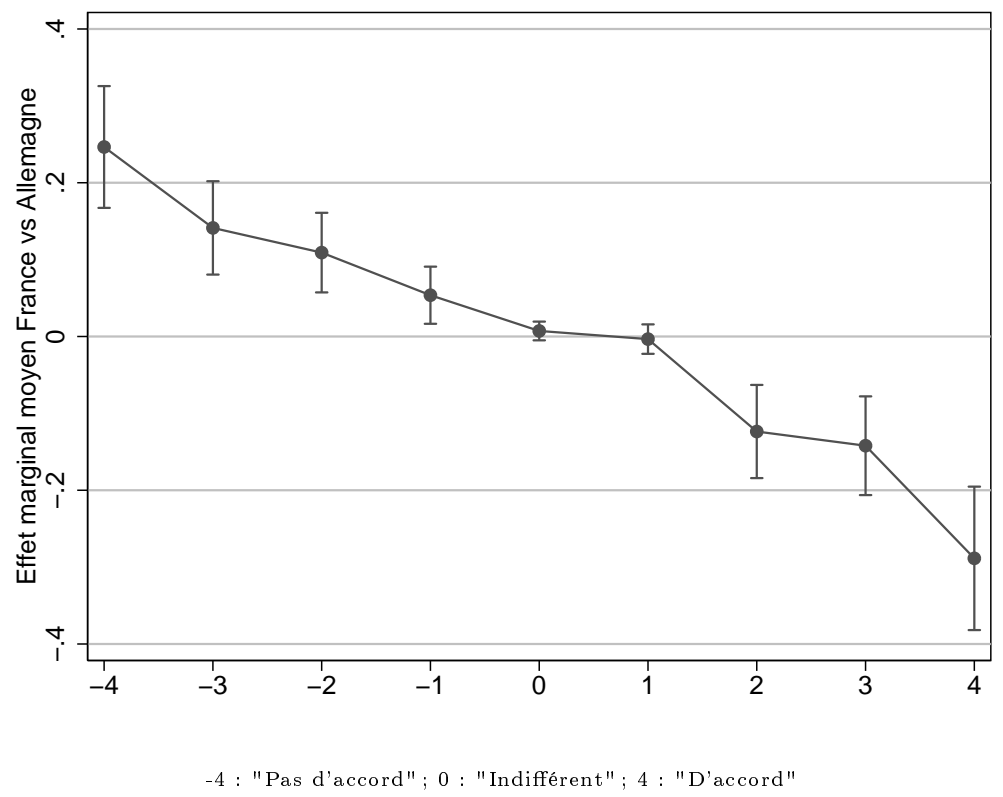

\section{Tests de robustesse}

Nous procédons à quatre tests de robustesse. Ces analyses présentent des résultats en ligne avec les conclusions de notre analyse principale. Les résultats principaux sont donnés dans 
cette section (voir l'Annexe C pour plus de détails).

\subsection{Modèle de sélection de Heckman}

Nous contrôlons ici pour un éventuel biais de sélection dû au fait que la participation à l'enquête s'est faite sur la base du volontariat. En effet, en dehors de questions de temps ou de manque de compétences sur les questions européennes, il est possible que l'absence de réponse révèle une opinion non exprimée sur ces questions. Dans ce cas, l'échantillon que nous utilisons ne serait pas représentatif des opinions des parlementaires.

Nous utilisons comme variables instrumentales le fait de participer ou non à la Commission des Affaires Européennes et dans celle des Finances. Cette variable binaire a un effet positif et significativement différent de zéro sur la participation à l'enquête. Elle n'en a cependant pas sur les réponses données. Nous procédons ensuite à l'estimation d'un modèle Probit binaire avec comme première étape le modèle de sélection de Heckman (voir Heckman (1979)).

Nous trouvons des résultats en ligne avec ceux donnés dans la section suivante (4.2, Probit binaire) avec pour exception la question portant sur le marché du travail, qui met en avant une différence significative entre les groupes S\&D et PPE, mais à $90 \%$ seulement. Cela suggère qu'il y a un biais de sélection pour cette question, pour laquelle les parlementaires les plus modérés de part et d'autre du spectre politique n'auraient pas participé.

\subsection{Probit binaire}

Nous construisons une variable binaire qui prend pour valeur 1 si le/la parlementaire est d'accord ou indifférent(e) et 0 si il/elle n'est pas d'accord avec la proposition. Cela permet une comparaison entre opposition forte et préférence faible pour chaque question en plus d'un test de robustesse.

Les résultats obtenus (voir Table 15) confirment ceux que nous avons trouvé dans la section précédente, à l'exception de la question concernant une assurance chômage européenne commune où l'on ne trouve plus d'effet significatif pour la nationalité. Cela est cependant cohérent avec le faible effet estimé dans le modèle Probit ordonné. Les effets relatifs sont également en ligne avec nos résultats.

Ces résultats tendent à confirmer deux potentiels terrains d'entente franco-allemand mis en lumière dans l'analyse préliminaire : la flexibilité du marché du travail et une assurance chômage européenne commune. 


\subsection{Définitions plus larges des partis}

Nous prenons ici une définition plus large de l'appartenance politique en définissant comme "gauche large" un groupe comprenant les groupes S\&D, Verts/ALE et GUE/NGL, et comme "centre droit large" un groupe comprenant les groupes PPE, ALDE et ENL.

Les résultats sont alignés avec ceux trouvés dans l'analyse principale, avec des ordres de grandeur très similaires. La nationalité a cependant un effet moins significatif et moins important en ce qui concerne la question sur la politique d'immigration.

\subsection{Par nationalité et par parti}

Ce test consiste à estimer le modèle Probit ordonné :

- en France puis en Allemagne (considérer l'effet de l'appartenance politique dans chaque pays séparément)

- au sein du groupe S\&D puis du groupe PPE (considérer l'effet de la nationalité dans chaque groupe séparément).

Chacune des questions fait ressortir une division idéologique pour la France et pour l'Allemagne, sauf celle de l'immigration, qui ne montre pas d'effet significatif de l'appartenance politique en Allemagne.

De même, on trouve un effet significatif de la nationalité dans les groupes S\&D et PPE pour les questions relatives au marché du travail et à la politique salariale, et seulement dans le groupe PPE pour les questions relatives à l'assurance chômage européenne et l'immigration. Sur la flexibilité du marché du travail, nous n'obtenons pas d'effet de la nationalité.

\subsection{Hétéroscédasticité}

En prenant en compte une potentielle hétéroscédasticité induite par les variables liées à la nationalité et à l'appartenance politique, nous trouvons des effets allant dans le même sens que dans le modèle estimé sous hypothèse d'homoscédasticité. De même, la seule question pour laquelle la nationalité n'a pas d'effet significativement différent de zéro est celle portant sur la flexibilité du marché du travail.

\section{Conclusion}

Cet article présente les résultats de la première enquête réalisée auprès des parlementaires français et allemands sur l'avenir du projet européen dans le domaine du marché du travail. Nous montrons comment les réponses des parlementaires reflètent de potentiels terrains d'entente entre les deux pays, au-delà de clivages partisans. 
Alors que la création d'une assurance chômage européenne et la flexibilité du marché du travail révèlent un clivage gauche/droite plutôt que français/allemand, une politique d'immigration commune est globalement soutenue par les parlementaires des deux pays ainsi que des deux bords politiques. Les questions de directives contraignantes dans le marché du travail et de la politique salariale ne trouvent d'alignement ni des pays ni des partis. Le premier, cependant, fait ressortir un clivage partisan de moindre ampleur que dans le reste des questions.

Enfin, il ressort du fort clivage droite/gauche sur certaines questions que l'euroscepticisme observé dans plusieurs discours politiques pourrait dans certains cas relever d'une position idéologique plutôt que d'une opinion tranchée contre l'intégration européenne elle-même. 


\section{Annexes}

\section{A Caractéristiques individuelles}

\section{A.1 Échantillon total}

TABLE 8 - Nationalités et groupes politiques

\begin{tabular}{l|r} 
France & $59,4 \%$ \\
Allemagne & $40,6 \%$ \\
\hline Groupe S\&D & $39,7 \%$ \\
Groupe PPE & $41,9 \%$ \\
Autres & $18,4 \%$
\end{tabular}

TABle 9 - Caractéristiques des parlementaires

(a) Par nationalité

\begin{tabular}{l|cc} 
& $\begin{array}{c}\text { France } \\
(922)\end{array}$ & $\begin{array}{c}\text { Allemagne } \\
(630)\end{array}$ \\
\hline Groupe S\&D & $45,9 \%$ & $30,6 \%$ \\
Groupe PPE & $36,9 \%$ & $49,2 \%$ \\
Autres & $17,2 \%$ & $20,2 \%$ \\
Femmes & $25,8 \%$ & $36,7 \%$ \\
Baccalauréat & $88,5 \%$ & $82,6 \%$ \\
Études supérieures & $83,7 \%$ & $77,9 \%$ \\
Âge (moyenne) & 60,2 & 52,8 \\
Nombre d'années au parlement (moyenne) & 9,9 & 9 \\
\hline
\end{tabular}

(b) Par groupe politique

\begin{tabular}{l|ccc} 
& S\&D & PPE & Autres \\
& $(616)$ & $(650)$ & $(286)$ \\
\hline Parlementaires français & $68,7 \%$ & $52,3 \%$ & $55,6 \%$ \\
Parlementaires allemands & $31,3 \%$ & $47,7 \%$ & $44,4 \%$ \\
Femmes & $36,4 \%$ & $20 \%$ & $39,5 \%$ \\
Baccalauréat & $84,9 \%$ & $85,9 \%$ & $88,5 \%$ \\
Études supérieures & $81,3 \%$ & $81,6 \%$ & $80,4 \%$ \\
Âge (moyenne) & 57,9 & 57,3 & 55,4 \\
Nombre d'années au parlement (moyenne) & 8,9 & 10,7 & 8,5 \\
\hline
\end{tabular}




\section{A.2 Participants et non-participants}

TABLE 10 - Caractéristiques des parlementaires

\begin{tabular}{l|cc} 
& $\begin{array}{c}\text { Participants } \\
(232)\end{array}$ & $\begin{array}{c}\text { Non-participants } \\
(1320)\end{array}$ \\
\hline Parlementaires français & $56,4 \%$ & $59,9 \%$ \\
Parlementaires allemands & $43,6 \%$ & $40,1 \%$ \\
Groupe S\&D & $47,4 \%$ & $38,3 \%$ \\
Groupe PPE & $37,9 \%$ & $42,6 \%$ \\
Autres & $14,7 \%$ & $19,1 \%$ \\
Femmes & $27,2 \%$ & $30,8 \%$ \\
Baccalauréat & $88,3 \%$ & $85,5 \%$ \\
Études supérieures & $82,3 \%$ & $81,1 \%$ \\
Âge (moyenne) & 58,9 & 56,9 \\
Nombre d'années au parlement (moyenne) & 9 & 9,7 \\
\hline
\end{tabular}

\section{A.3 Parlementaires ayant répondu}

TABle 11 - Par nationalité

(a) Composition politique, sexe et études

\begin{tabular}{l|cc} 
& $\begin{array}{c}\text { France } \\
(131)\end{array}$ & $\begin{array}{c}\text { Allemagne } \\
(101)\end{array}$ \\
\hline Groupe S\&D & $54,2 \%$ & $38,6 \%$ \\
Groupe PPE & $35,9 \%$ & $40,6 \%$ \\
Autres & $9,9 \%$ & $20,8 \%$ \\
Femmes & $21,4 \%$ & $34,7 \%$ \\
Baccalauréat & $91,7 \%$ & $84,2 \%$ \\
Études supérieures & $90,1 \%$ & $72,7 \%$ \\
\hline
\end{tabular}

(b) Âge

\begin{tabular}{l|ccc} 
& Minimum & Maximum & Moyenne \\
\hline France & 35 & 76 & 61 \\
Allemagne & 33 & 81 & 56 \\
\hline Total & 33 & 81 & 59 \\
\hline
\end{tabular}

(c) Nombre d'années au parlement

\begin{tabular}{l|ccc} 
& Minimum & Maximum & Moyenne \\
\hline France & 1 & 35 & 9 \\
Allemagne & 1 & 40 & 9 \\
\hline Total & 1 & 40 & 9 \\
\hline
\end{tabular}


TABLE 12 - Par groupe politique

(a) Composition nationale, sexe et études

\begin{tabular}{l|ccc} 
& $\begin{array}{c}\text { S\&D } \\
(110)\end{array}$ & $\begin{array}{c}\text { PPE } \\
(88)\end{array}$ & $\begin{array}{c}\text { Autres } \\
(34)\end{array}$ \\
\hline Parlementaires français & $64,5 \%$ & $53,4 \%$ & $38,2 \%$ \\
Parlementaires allemands & $35,5 \%$ & $46,6 \%$ & $61,8 \%$ \\
Femmes & $31,8 \%$ & $18,2 \%$ & $35,3 \%$ \\
Baccalauréat & $87,6 \%$ & $88,1 \%$ & $90,9 \%$ \\
Études supérieures & $85,6 \%$ & $79,5 \%$ & $78,8 \%$ \\
\hline
\end{tabular}

(b) Âge

\begin{tabular}{l|ccc} 
& Minimum & Maximum & Moyenne \\
\hline S\&D & 35 & 76 & 59 \\
PPE & 33 & 81 & 60 \\
Autres & 43 & 73 & 57 \\
\hline Total & 33 & 81 & 59 \\
\hline
\end{tabular}

(c) Nombre d'années au parlement

\begin{tabular}{l|ccc} 
& Minimum & Maximum & Moyenne \\
\hline S\&D & 1 & 36 & 8 \\
PPE & 1 & 40 & 11 \\
Autres & 1 & 17 & 7,5 \\
\hline Total & 1 & 40 & 9 \\
\hline
\end{tabular}

\section{B Tests statistiques sur les réponses}

\section{B.1 Tests Chi2}

1. Marché du travail : p-valeur $<0,01$ pour la Nationalité et l'appartenance politique

2. Politique salariale : p-valeur $<0,01$ pour la Nationalité et l'appartenance politique

3. Politique d'immigration : p-valeur de 0,02 pour la Nationalité et de 0,026 pour l'appartenance politique

4. Assurance chômage européenne : p-valeur de 0,014 pour la Nationalité et $<0,01$ pour l'appartenance politique

5. Marché du travail plus flexible : p-valeur de 0,801 pour la Nationalité et $<0,01$ pour l'appartenance politique 


\section{B.2 Tests de Mann-Whitney}

TABLE 13 - Tests de Mann-Whitney : p-valeurs

\begin{tabular}{l|ccccc} 
& \multicolumn{5}{c}{ France/Allemagne } \\
& $\begin{array}{c}\text { Marché } \\
\text { du travail }\end{array}$ & $\begin{array}{c}\text { Politique } \\
\text { salariale }\end{array}$ & Immigration & $\begin{array}{c}\text { Assurance } \\
\text { chômage }\end{array}$ & $\begin{array}{c}\text { Flexibilité du } \\
\text { marché du travail }\end{array}$ \\
\hline Test de rang & $<0,01$ & $<0,01$ & $<0,01$ & $<0,01$ & 0,397 \\
Test de médiane & $<0,01$ & $<0,01$ & 0,864 & 0,129 & 0,397 \\
\hline
\end{tabular}

\begin{tabular}{l|ccccc} 
& \multicolumn{9}{c}{$\mathrm{S} \& \mathrm{D} / \mathrm{PPE}$} & & \\
& $\begin{array}{c}\text { Marché } \\
\text { du travail }\end{array}$ & $\begin{array}{c}\text { Politique } \\
\text { salariale }\end{array}$ & Immigration & $\begin{array}{c}\text { Assurance } \\
\text { chômage }\end{array}$ & $\begin{array}{c}\text { Flexibilité du } \\
\text { marché du travail }\end{array}$ \\
\hline Test de rang & $<0,01$ & $<0,01$ & $<0,01$ & $<0,01$ & $<0,01$ \\
Test de médiane & $<0,01$ & $<0,01$ & 0,014 & $<0,01$ & $<0,01$ \\
\hline
\end{tabular}

\section{B.3 Tests de proportions}

TABLE 14 - Proportion des parlementaires en faveur de la proposition

\begin{tabular}{|c|c|c|c|c|c|c|}
\hline & & $\begin{array}{c}\text { Marché } \\
\text { du travail }\end{array}$ & $\begin{array}{l}\text { Politique } \\
\text { salariale }\end{array}$ & Immigration & $\begin{array}{l}\text { Assurance } \\
\text { chômage }\end{array}$ & $\begin{array}{c}\text { Flexibilité du } \\
\text { marché du travail }\end{array}$ \\
\hline \multirow{2}{*}{ France } & proportion (pr) & 0,69 & 0,75 & 0,72 & 0,58 & 0,51 \\
\hline & $\mathrm{p}$-valeur $(\mathrm{H} 0: \mathrm{pr}=0,5)$ & $<0,01$ & $<0,01$ & $<0,01$ & 0,11 & 0,26 \\
\hline \multirow{2}{*}{ Allemagne } & proportion (pr) & 0,40 & 0,33 & 0,93 & 0,45 & 0,44 \\
\hline & $\mathrm{p}$-valeur $(\mathrm{Ho}: \mathrm{pr}=0,5)$ & 0,05 & $<0,01$ & $<0,01$ & 0,39 & 0,86 \\
\hline
\end{tabular}

Note : une p-valeur supérieure à 0,1 indique que l'on ne peut pas rejeter H0, c'est-à-dire que la proportion de parlementaires en faveur de la proposition n'est pas statistiquement différente de 0,5 .

\section{Robustesse}

\section{C.1 Modèle de sélection de Heckman}

1. Marché du travail :

- Effet positif et significatif à $99 \%$ de la nationalité.

- Effet positif et significatif à 90\% de l'appartenance politique. 
2. Politique salariale :

- Effet positif et significatif à $99 \%$ de la nationalité.

- Effet positif et significatif à $99 \%$ de l'appartenance politique.

3. Politique d'immigration :

- Effet négatif et significatif à $95 \%$ de la nationalité.

- Effet positif et significatif à $99 \%$ de l'appartenance politique.

4. Assurance chômage européenne :

- Pas d'effet statistiquement significatif de la nationalité.

- Effet positif et significatif à 99\% de l'appartenance politique.

5. Marché du travail plus flexible :

- Pas d'effet statistiquement significatif de la nationalité.

- Effet négatif et significatif à 99\% de l'appartenance politique.

\section{C.2 Probit binaire}

Les résultats obtenus sont donnés dans la table d'effets marginaux moyens ci-dessous.

TABle 15 - Probit binaire : Effets marginaux moyens

\begin{tabular}{|c|c|c|c|c|c|}
\hline & $\begin{array}{c}\text { Marché } \\
\text { du travail }\end{array}$ & $\begin{array}{c}\text { Politique } \\
\text { salariale }\end{array}$ & Immigration & $\begin{array}{c}\text { Assurance } \\
\text { chômage }\end{array}$ & $\begin{array}{c}\text { Flexibilité du } \\
\text { marché du travail }\end{array}$ \\
\hline $\mathrm{Fr}$ & $\begin{array}{c}0.25^{* * *} \\
(0.074)\end{array}$ & $\begin{array}{c}0.33^{* * *} \\
(0.071)\end{array}$ & $\begin{array}{c}-0.20 * * * \\
(0.056)\end{array}$ & $\begin{array}{c}0.11 \\
(0.073)\end{array}$ & $\begin{array}{c}0.024 \\
(0.062)\end{array}$ \\
\hline$S \& D$ & $\begin{array}{l}0.18^{* *} \\
(0.072)\end{array}$ & $\begin{array}{c}0.25^{* * *} \\
(0.069)\end{array}$ & $\begin{array}{c}0.22 * * * \\
(0.060)\end{array}$ & $\begin{array}{c}0.40 * * * \\
(0.073)\end{array}$ & $\begin{array}{c}-0.64^{* * *} \\
(0.058)\end{array}$ \\
\hline Femme & $\begin{array}{c}0.14^{*} \\
(0.080)\end{array}$ & $\begin{array}{c}0.093 \\
(0.077)\end{array}$ & $\begin{array}{c}0.083 \\
(0.071)\end{array}$ & $\begin{array}{l}-0.040 \\
(0.085)\end{array}$ & $\begin{array}{c}0.017 \\
(0.074)\end{array}$ \\
\hline Age & $\begin{array}{c}0.0060 \\
(0.0038)\end{array}$ & $\begin{array}{c}0.011^{* * *} \\
(0.0034)\end{array}$ & $\begin{array}{l}-0.0025 \\
(0.0035)\end{array}$ & $\begin{array}{c}0.0011 \\
(0.0041)\end{array}$ & $\begin{array}{c}0.00035 \\
(0.0036)\end{array}$ \\
\hline Annees & $\begin{array}{c}-0.011^{* *} \\
(0.0046)\end{array}$ & $\begin{array}{c}-0.012^{* * *} \\
(0.0043)\end{array}$ & $\begin{array}{c}0.0036 \\
(0.0043)\end{array}$ & $\begin{array}{l}-0.0030 \\
(0.0049)\end{array}$ & $\begin{array}{l}-0.0010 \\
(0.0043)\end{array}$ \\
\hline $\mathrm{Bac}$ & $\begin{array}{c}-0.25^{* *} \\
(0.12)\end{array}$ & $\begin{array}{l}-0.25^{*} \\
(0.13)\end{array}$ & $\begin{array}{c}0.0096 \\
(0.16)\end{array}$ & $\begin{array}{l}-0.11 \\
(0.18)\end{array}$ & $\begin{array}{l}-0.035 \\
(0.17)\end{array}$ \\
\hline Etudes & $\begin{array}{c}0.32^{* *} \\
(0.16)\end{array}$ & $\begin{array}{l}0.27^{*} \\
(0.16)\end{array}$ & $\begin{array}{l}0.039 \\
(0.16)\end{array}$ & $\begin{array}{c}0.14 \\
(0.18)\end{array}$ & $\begin{array}{l}0.088 \\
(0.15)\end{array}$ \\
\hline Observations & 183 & 182 & 185 & 182 & 182 \\
\hline
\end{tabular}


1. Marché du travail :

- Effet positif et significatif à $99 \%$ de la nationalité : ceteris paribus, un/une parlementaire français(e) a en moyenne une probabilité de $25 \%$ supérieure à un/une parlementaire allemand(e) d'être en faveur ou indifférent(e) à cette proposition.

- Effet positif et significatif à 95\% de l'appartenance politique : ceteris paribus, un/une parlementaire du groupe S\&D a en moyenne une probabilité de $18 \%$ supérieure à un/une parlementaire du groupe PPE d'être en faveur ou indifférent(e) à cette proposition.

2. Politique salariale :

- Effet positif et significatif à $99 \%$ de la nationalité : ceteris paribus, un/une parlementaire français(e) a en moyenne une probabilité de $33 \%$ supérieure à un/une parlementaire allemand(e) d'être en faveur ou indifférent(e) à cette proposition.

- Effet positif et significatif à 99\% de l'appartenance politique : ceteris paribus, un/une parlementaire du groupe S\&D a en moyenne une probabilité de $25 \%$ supérieure à un/une parlementaire du groupe PPE d'être en faveur ou indifférent(e) à cette proposition.

3. Politique d'immigration :

- Effet négatif et significatif à $99 \%$ de la nationalité : ceteris paribus, un/une parlementaire allemand(e) a en moyenne une probabilité de $20 \%$ supérieure à un/une parlementaire français(e) d'être en faveur ou indifférent(e) à cette proposition.

- Effet positif et significatif à $99 \%$ de l'appartenance politique : ceteris paribus, un/une parlementaire du groupe S\&D a en moyenne une probabilité de $22 \%$ supérieure à un/une parlementaire du groupe PPE d'être en faveur ou indifférent(e) à cette proposition.

4. Assurance chômage européenne :

- Effet positif et significatif à $99 \%$ de l'appartenance politique : ceteris paribus, un/une parlementaire du groupe S\&D a en moyenne une probabilité de $40 \%$ supérieure à un/une parlementaire du groupe PPE d'être pour ou indifférent à cette proposition

5. Marché du travail plus flexible : 
- Effet négatif et significatif à $99 \%$ de l'appartenance politique : ceteris paribus, un/une parlementaire du groupe PPE a en moyenne une probabilité de $64 \%$ supérieure à un/une parlementaire du groupe S\&D d'être en faveur ou indifférent(e) à cette proposition

\section{C.3 Définition plus large des partis}

Nous trouvons les résultats suivants. Les tables sont disponibles sur demande.

1. Marché du travail :

- Effet positif et significatif à $99 \%$ de la nationalité.

- Effet positif et significatif à $99 \%$ de l'appartenance politique.

2. Politique salariale :

- Effet positif et significatif à $99 \%$ de la nationalité.

- Effet positif et significatif à $99 \%$ de l'appartenance politique.

3. Politique d'immigration :

- Effet négatif et significatif à $90 \%$ de la nationalité.

- Effet positif et significatif à $99 \%$ de l'appartenance politique.

4. Assurance chômage européenne :

- Effet positif et significatif à $99 \%$ de la nationalité.

- Effet positif et significatif à $99 \%$ de l'appartenance politique.

5. Marché du travail plus flexible :

- Effet négatif et significatif à 99\% de l'appartenance politique.

\section{C.4 Par nationalité et par parti}

Nous trouvons les résultats suivants. Les tables sont disponibles sur demande.

1. Marché du travail :

- Effets positifs et significatifs à un niveau de $99 \%$ de l'appartenance politique en France et en Allemagne. 
- Effets positifs et significatifs à un niveau de $99 \%$ de la nationalité dans les groupes S\&D et PPE.

2. Politique salariale :

- Effets positifs et significatifs à un niveau de $99 \%$ de l'appartenance politique en France et en Allemagne.

- Effets positifs et significatifs à un niveau de $99 \%$ de la nationalité dans les groupes S\&D et PPE.

3. Politique d'immigration :

- Effet positif et significatif à un niveau de $99 \%$ de l'appartenance politique en France; pas d'effet significatif de l'appartenance politique en Allemagne.

- Effet négatif et significatif à un niveau de $95 \%$ de la nationalité dans le groupe PPE. Pas d'effet significatif dans le groupe S\&D.

4. Assurance chômage européenne :

- Effets positifs et significatifs à un niveau de $99 \%$ de l'appartenance politique en France et en Allemagne.

- Effets positifs et significatifs à un niveau de $95 \%$ de la nationalité dans le groupe PPE. Pas d'effet significatif dans le groupe S\&D.

5. Marché du travail plus flexible :

- Effets négatifs et significatifs à un niveau de $99 \%$ de l'appartenance politique en France et en Allemagne.

- Pas d'effet significatif, ni dans le groupe S\&D ni dans le groupe PPE.

\section{C.5 Hétéroscédasticité}

Nous supposons ici une variance exponentielle et nous obtenons les résultats suivants (les tables sont disponibles sur demande) :

1. Marché du travail :

- Effet positif et significatif à $99 \%$ de la nationalité.

- Effet positif et significatif à 99\% de l'appartenance politique.

2. Politique salariale : 
- Effet positif et significatif à $99 \%$ de la nationalité.

- Effet positif et significatif à $99 \%$ de l'appartenance politique.

3. Politique d'immigration :

- Effet négatif et significatif à $99 \%$ de la nationalité.

- Effet positif et significatif à $99 \%$ de l'appartenance politique.

4. Assurance chômage européenne :

- Effet positif et significatif à $95 \%$ de la nationalité.

- Effet positif et significatif à $99 \%$ de l'appartenance politique.

5. Marché du travail plus flexible :

- Effet négatif et significatif à $99 \%$ de l'appartenance politique. 


\section{Questionnaire}

\section{Questionnaire : De l'avenir de l'Union Européenne}

\section{Répartition des missions et des compétences en Europe}

Actuellement l'élargissement des compétences de I'UE dans certains domaines politiques est en débat. Approuvez-vous les propositions suivantes?

\section{Politique énergétique}

L'UE pourrait donner des directives contraignantes aux États membres quant à leur choix de mix énergétique dans leur production d'électricité (par exemple en fixant la proportion des énergies renouvelables, du charbon, et de l'énergie nucléaire).

Pas d'accord

Indifférent

D'accord

\begin{tabular}{l|l|l|l|l|l|l|l|l|l}
$\square-4$ & $\square-3$ & $\square-2$ & $\square-1$ & $\square 0$ & $\square+1$ & $\square+2$ & $\square+3$ & $\square+4$
\end{tabular}

\section{Politique d'Immigration}

L'UE devrait jouer un rôle renforcé dans la politique d'immigration des États membres (par exemple en fixant les normes standard d'accueil ou en décidant de la répartition des réfugiés).

Pas d'accord

Indifférent

D'accord

\begin{tabular}{l|l|l|l|l|l|l|l|l|l}
$\square-4$ & $\square-3$ & $\square-2$ & $\square-1$ & $\square 0$ & $\square+1$ & $\square+2$ & $\square+3$ & $\square+4$
\end{tabular}

\section{Politique fiscale}

Le Conseil européen pourrait statuer avec une majorité qualifiée sur les impôts perçus dans les États membres

(par exemple sur les limites minimales ou maximales obligatoires des impôts sur les Sociétés).

Pas d'accord

Indifférent

D'accord

\begin{tabular}{l|l|l|l|l|l|l|l|l|l|l|}
$\square-4$ & $\square-3$ & $\square-2$ & $\square-1$ & $\square 0$ & $\square+1$ & $\square+2$ & $\square+3$ & $\square+4$
\end{tabular}

\section{Politique salariale}

L'UE pourrait avoir des droits d'intervention plus importants dans les politiques salariales des États membres (par exemple sur le niveau du salaire minimum légal).

Pas d'accord

Indifférent

D'accord

\begin{tabular}{l|l|l|l|l|l|l|l|l|l|l}
$\square-4$ & $\square-3$ & $\square-2$ & $\square-1$ & $\square 0$ & $\square+1$ & $\square+2$ & $\square+3$ & $\square+4$
\end{tabular}

\section{Marché du travail}

L'UE pourrait donner des directives contraignantes aux États membres relatives au marché du travail (par exemple sur les modalités de la protection des salariés contre les licenciements ou sur les clauses du contrat de travail à durée déterminée).

Pas d'accord

Indifférent

D'accord

\begin{tabular}{l|l|l|l|l|l|l|l|l|l}
$\square-4$ & $\square-3$ & $\square-2$ & $\square-1$ & $\square 0$ & $\square+1$ & $\square+2$ & $\square+3$ & $\square+4$
\end{tabular}

\section{Politique de Défense}

L'UE pourrait constituer une armée européenne placée sous son commandement et financée par son budget avec pour mission d'assurer les opérations extérieures à la place des armées nationales.

Pas d'accord

Indifférent

D'accord

\begin{tabular}{|l|l|l|l|l|l|l|l|l|}
$\square-4$ & $\square-3$ & $\square-2$ & $\square-1$ & $\square 0$ & $\square+1$ & $\square+2$ & $\square+3$ & $\square+4$
\end{tabular}




\section{Pacte budgétaire européen}

Le pacte budgétaire européen ratifié en 2012 par 25 États de l'UE engage les États signataires, comme la France et l'Allemagne, à limiter leur déficit public (corrigé des variations conjoncturelles) à $0,5 \%$ du produit intérieur brut.

Pensez-vous que la France devrait respecter les exigences du pacte budgétaire européen ?

Pas d'accord

Indifférent

D'accord

$\square-4$

$-3$

$-2$

$\square-1$

$\square 0$

$+1$

$+2$

$\square+3$

$\square+4$

Pensez-vous que les États suivants de la zone euro respectent régulièrement les exigences du pacte budgétaire européen? (réponses multiples possibles)

\begin{tabular}{|c|c|c|c|c|c|c|c|}
\hline Pays & Oui & Non & $\begin{array}{l}\text { Je ne } \\
\text { sais pas }\end{array}$ & Pays & Oui & Non & $\begin{array}{l}\text { Je ne } \\
\text { sais pas }\end{array}$ \\
\hline Allemagne & & & & Italie & & & \\
\hline Autriche & & & & Lettonie & & & \\
\hline Belgique & & & & Lituanie & & & \\
\hline Chypre & & & & Luxembourg & & & \\
\hline Espagne & & & & Malte & & & \\
\hline Estonie & & & & Pays-Bas & & & \\
\hline Finlande & & & & Portugal & & & \\
\hline France & & & & Slovaquie & & & \\
\hline Grèce & & & & Slovénie & & & \\
\hline Irlande & & & & & & & \\
\hline
\end{tabular}




\section{Politique monétaire et des finances dans la zone euro}

La politique monétaire et des finances dans la zone euro fait l'objet de points de vue divergents. Approuvez-vous les propositions suivantes?

Investissements plus élevés

Une augmentation des dépenses d'investissements des États membres est indispensable pour une croissance plus forte dans la zone euro.

Pas d'accord

Indifférent

D'accord

$\square-4$

$-3$

$-2$

$\square-1$

$\square 0$

$\square+1$

$\square+2$

$\square+3$

$\square+4$

\section{Marché du travail plus flexible}

Une croissance plus forte dans la zone euro requière que les États comptabilisant un nombre important de chômeurs de longue durée rendent plus flexible leur marché du travail

(par exemple en assouplissant les conditions de licenciement ou en baissant le niveau du salaire minimum).

Pas d'accord Indifférent D'accord

\begin{tabular}{l|l|l|l|l|l|l|l|l|l}
$\square-4$ & $\square-3$ & $\square-2$ & $\square-1$ & $\square 0$ & $\square+1$ & $\square+2$ & $\square+3$ & $\square+4$
\end{tabular}

\section{Assurance chômage européenne}

Pour pallier une éventuelle récession de certains États membres dans la zone euro il faudrait créer une assurance chômage commune européenne.

\begin{tabular}{c|c|c|c|c|c|c|c|c} 
Pas d'accord & Indifférent \\
\hline-4 & $\square-3$ & $\square-2$ & $\square-1$ & $\square 0$ & $\square+1$ & $\square+2$ & $\square+3$ & $\square+4$
\end{tabular}

\section{Euro-obligations}

La zone euro devrait émettre des euro-obligations et les États membres s'en porter tous garants solidairement et bénéficier du même taux d'intérêt.

Pas d'accord Indifférent D'accord

\begin{tabular}{l|l|l|l|ll|l|l|l|l|}
$\square-4$ & $\square-3$ & $\square-2$ & $\square-1$ & $\square 0$ & $\square+1$ & $\square+2$ & $\square+3$ & $\square+4$
\end{tabular}

\section{Achats d'emprunts par la BCE}

Pour combattre la crise, la Banque centrale européenne s'est engagée fortement dans les années passées en achetant des emprunts d'États de la zone euro. Cet engagement devrait se poursuivre.

Pas d'accord

Indifférent

D'accord

\begin{tabular}{l|l|l|l|l|l|l|l|l|l}
$\square-4$ & $\square-3$ & $\square-2$ & $\square-1$ & $\square 0$ & $\square+1$ & $\square+2$ & $\square+3$ & $\square+4$
\end{tabular}




\section{Références}

[1] Alesina, A., Angeloni, I. et Shuknecht, L. (2005). What Does the European Union Do? Public Choice 123 : 275-319.

[2] Blesse, S., Boyer, P.C., Heinemann, F., et Janeba, E. (2016). Searching for a FrancoGerman consensus on the future of Europe : Survey results for Bundestag, Assemblée Nationale and Sénat. ZEW policy brief, No. 5/2016.

[3] Boeri, T., Conde-Ruiz, J.I., et Galasso, V. (2012). The political economy of flexicurity. Journal of the European Economic Association, 10(4), 684-715.

[4] Cahuc, P., Carcillo, S. et Zylberberg, A. (2014). Labor economics (2nd Edition). MIT Press.

[5] Dolls, M., Fuest, F., Neumann, D. et Peichl, A. (à paraître), An Unemployment Insurance Scheme for the Euro Area? A Comparison of Different Alternatives using Micro Data, International Tax and Public Finance.

[6] Farhi, E., et Werning, I. (2014). Labor mobility within currency unions. National Bureau of Economic Research (No. w20105).

[7] Heckman, J. (1979). Sample selection bias as a specification error. Econometrica, 47(1), 153-161.

[8] Oates, W.E. (1999). An essay on fiscal federalism. Journal of economic literature, 37(3), 1120-1149.

[9] Rosanvallon, P. (2013). La légitimité démocratique : impartialité, réflexivité, proximité. Seuil.

[10] Saint-Paul, G. (1996). Exploring the political economy of labour markets institutions. Economic Policy, 11(23), 263-315.

[11] Saint-Paul, G. (2002). The political economy of employment protection. Journal of political economy, 110(3), 672-704.

[12] Turrini, A., Koltay, G., Pierini, F., Goffard, C., et Kiss, A. (2015). A decade of labour market reforms in the EU : insights from the LABREF database. IZA Journal of Labor Policy, 4(1), 1. 